\title{
Stellar substructures in the periphery of the Magellanic Clouds with the VISTA Hemisphere Survey from the red clump and other tracers
}

\author{
Dalal El Youssoufi, ${ }^{1 \star}$ Maria-Rosa L. Cioni, ${ }^{1}$ Cameron P. M. Bell, ${ }^{1}$ \\ Florian Niederhofer, ${ }^{1}$ Joana M. Oliveira, ${ }^{7}$ Vincenzo Ripepi,${ }^{8}$ Thomas Schmidt, ${ }^{1}$ \\ Smitha Subramanian, ${ }^{9}$ Ning-Chen Sun, ${ }^{10}$ and Jacco Th. van Loon ${ }^{7}$ \\ ${ }^{1}$ Leibniz-Institut für Astrophysik Potsdam (AIP), An der Sternwarte 16, D-14482 Potsdam, Germany \\ ${ }^{2}$ Department of Physics and Astronomy, Macquarie University, Balaclava Road, Sydney, NSW 2109, Australia \\ ${ }^{3}$ Research Centre for Astronomy, Astrophysics and Astrophotonics, Macquarie University, Balaclava Road, Sydney, NSW 2109, Australia \\ ${ }^{4}$ International Space Science Institute-Beijing, 1 Nanertiao, Zhongguancun, Hai Dian District, Beijing 100190, China \\ ${ }^{5}$ Koninklijke Sterrenwacht van België, Ringlaan 3, B-1180 Brussels, Belgium \\ ${ }^{6}$ European Southern Observatory, Karl-Schwarzschild-Str. 2, D-85748 Garching bei München, Germany \\ ${ }^{7}$ Lennard-Jones Laboratories, Keele University, ST5 5BG, UK \\ ${ }^{8}$ INAF - Osservatorio Astronomico di Capodimonte, via Moiariello 16, I-80131, Naples, Italy \\ ${ }^{9}$ Indian Institute of Astrophysics, Koramangala II Block, Bangalore-34, India \\ ${ }^{10}$ Department of Physics and astronomy, University of Sheffield, Hicks Building, Hounsfield Road, Sheffield S3 7RH, UK
}

Richard de Grijs, ${ }^{2,3,4}$ Martin A. T. Groenewegen, ${ }^{5}$ Valentin D. Ivanov, ${ }^{6}$ Gal Matijevič,${ }^{1}$

Accepted 2021 April 12. Received 2021 March 20; in original form 2020 August 15

\begin{abstract}
We study the morphology of the stellar periphery of the Magellanic Clouds in search of substructure using near-infrared imaging data from the VISTA Hemisphere Survey (VHS),Based on the selection of different stellar populations using the $\left(J-K_{\mathrm{s}}, K_{\mathrm{S}}\right)$ colour-magnitude diagram, we confirm the presence of substructures related to the interaction history of the Clouds and find new substructures on the eastern side of the LMC disc which may be owing to the influence of the Milky Way, and on the northern side of the SMC, which is probably associated to the ellipsoidal structure of the galaxy. We also study the luminosity function of red clump stars in the SMC and confirm the presence of a bi-modal distance distribution, in the form of a foreground population. We find that this bi-modality is still detectable in the eastern regions of the galaxy out to a $10^{\circ}$ distance from its centre. Additionally, a background structure is detected in the North between $7^{\circ}$ and $10^{\circ}$ from the centre which might belong to the Counter Bridge, and a foreground structure is detected in the South between $6^{\circ}$ and $8^{\circ}$ from the centre which might be linked to the Old Bridge.
\end{abstract}

Key words: Magellanic Clouds - galaxies: photometry - galaxies: interactions - galaxies: stellar content

\section{INTRODUCTION}

The Large Magellanic Cloud (LMC) and the Small Magellanic Cloud (SMC) are the closest pair of interacting dwarf galaxies to the Milky Way (MW). Located at distances of $50 \mathrm{kpc}$ (e.g. de Grijs \& Bono 2014a) and $60 \mathrm{kpc}$ (e.g. de Grijs \& Bono 2015), respectively, they represent important testbeds for fundamental astrophysical processes as they provide insights into resolved stellar populations, the cosmological distance scale, the interstellar medium, as well as galaxy interactions and morphology. The LMC is a barred Magellanic spiral galaxy, showing an asymmetric bar (e.g. Zhao \& Evans 2000), a few spiral arms (e.g. de Vaucouleurs \& Freeman 1972; El Youssoufi et al. 2019)

^ E-mail: delyoussoufi@aip.de (DE) and an inclined disc (e.g. Cioni et al. 2001; van der Marel 2001) with a warp (e.g. Olsen \& Salyk 2002; Choi et al. 2018a), while the SMC is an irregular dwarf galaxy characterised by a large line-of-sight depth (e.g. Subramanian \& Subramaniam 2012; Jacyszyn-Dobrzeniecka et al. 2017; Scowcroft et al. 2016; Ripepi et al. 2017; Muraveva et al. 2018). It has been found that stellar populations of different ages in the Magellanic Clouds (MCs) display different morphologies (e.g. Cioni et al. 2000; Nikolaev \& Weinberg 2000; Zaritsky et al. 2000; El Youssoufi et al. 2019). Additionally, the Magellanic system includes the Magellanic Bridge, a feature connecting the two Clouds that is most prominent in H I gas (Hindman et al. 1963) with young (e.g. Shapley 1940; Harris 2007; Skowron et al. 2014; Noël et al. 2015; Mackey et al. 2017) as well as intermediate-age and old stars associated with it (e.g. Bagheri et al. 2013; Carrera et al. 
2017; Jacyszyn-Dobrzeniecka et al. 2017). It also includes the Magellanic Stream, a $\sim 200^{\circ}$ long stream of gas trailing the Clouds in their orbit around the MW (Mathewson et al. 1974) without an identified stellar component as well as the Leading Arm (Putman et al. 1998), a gaseous feature extending beyond the Galactic plane (Nidever et al. 2010), and across the Galactic disc (McClure-Griffiths et al. 2008).

Dynamical interactions have shaped the evolutionary history of the MCs. The LMC and SMC have been dynamically coupled since at least $\sim 2 \mathrm{Gyr}$ ago and experienced a significant interaction $\sim 150$ Myr ago (e.g. Diaz \& Bekki 2012; Zivick et al. 2019). Two mechanisms, tidal and ram pressure stripping, have been suggested to account for the creation of the Magellanic Stream and Bridge (e.g. Besla et al. 2007; Diaz \& Bekki 2012; Salem et al. 2015). Dynamical interactions can explain many of the diffuse stellar substructures, including arcs, clumps and overdensities, discovered in the periphery of the MCs.

In the outskirts of the SMC ( $8^{\circ}$ from its centre), Pieres et al. (2017) found a stellar overdensity, named SMCNOD, using imaging data from the Dark Energy Survey (DES; Abbott et al. 2018). Subsequently, imaging data from the MAGellanic SatelLITEs Survey (MagLiteS; Drlica-Wagner et al. 2016) was used to study SMCNOD finding that it lies at the same distance as the SMC and contains mostly intermediate-age stars (6 Gyr old) with a small fraction of young stars (1 Gyr old). SMCNOD was most likely tidally stripped from the SMC. Martínez-Delgado et al. (2019) revisited a shell-like overdensity in the outskirts of the SMC using the Survey of the MAgellanic Stellar History (SMASH; Nidever et al. 2017) data and found that it is solely composed of young stars ( $\sim 150 \mathrm{Myr}$ old $)$. There is no evidence of it being of tidal origin and it may have formed in a recent star formation event resulting from an interaction with the LMC or the MW. Massana et al. (2020), also using SMASH data, discovered a new faint feature located at $14^{\circ}$ from the centre of the SMC, possibly associated with a more distant structure.

In the periphery of the LMC, Mackey et al. $(2016,2018)$ found numerous substructures in and around the galaxy, including an arclike structure $13.5^{\circ}$ North of the centre, of which the stellar populations are indistinguishable from those of the LMC disc. The stars in this feature were most likely stripped from the LMC due to either the tidal force of the MW or to an interaction with the SMC. Belokurov \& Erkal (2019) used Gaia DR2 to look for low surface brightness features in the periphery of the MCs and found two thin and long stellar streams in the northern and southern regions of the LMC. Numerical simulations indicate that the effect of the MW and SMC on the LMC are both important for the creation of tidal features. Using a combination of Gaia data release \#1 (DR1; Gaia Collaboration et al. 2016) and Galaxy Evolution Explorer (GALEX, Martin et al. 2005) imaging data, Belokurov et al. (2017) demonstrated that the distribution of RR Lyrae stars traces a bridge joining the SMC from its trailing tidal tail to the LMC. However, the nature of this bridge is still under debate as it has not been detected in OGLE (Jacyszyn-Dobrzeniecka et al. 2017, 2020b) nor in Gaia DR2 data (Clementini et al. 2019). Deason et al. (2017) found that Mira-like stars trace the LMC as far as $20^{\circ}$ from its centre.

Established tracers of substructures within and around the Magellanic Clouds are red clump (RC) stars, low mass stars which have undergone the helium flash to ignite helium burning in their cores (e.g. Girardi 2016 and references therein). Owing to their constrained location in the colour-magnitude diagram (CMD), they are used as probes of stellar distances allowing the study of the three-dimensional (3D) structure and reddening of the MCs (e.g. Subramanian \& Subramaniam 2010, 2012, 2013; Tatton et al. 2013; Choi et al. 2018a,b). Nidever et al. (2013) discovered a strong distance bi-modality in the luminosity function of RC stars with one component at $\sim 67 \mathrm{kpc}$ and a second component at $\sim 55 \mathrm{kpc}$ in three eastern fields located $4^{\circ}$ from the SMC centre. They suggested that the component found at $\sim 55 \mathrm{kpc}$ is the tidally stripped stellar counterpart of the Magellanic Bridge, in good agreement with dynamical models (e.g. Diaz \& Bekki 2012). Using the VISTA near-infrared $Y J K_{\mathrm{S}}$ survey of the MCs system (VMC; Cioni et al. 2011), Subramanian et al. (2017) studied the luminosity function of RC stars in the inner $20 \mathrm{deg}^{2}$ region of the SMC. A bi-modality in distances to the RC stars was found in the eastern SMC, after having ruled out reddening, RC population effects, and line of sight depth effects as potential causes, it was interpreted as a foreground population $\approx 11 \mathrm{kpc}$ in front of the main body. This is most likely due to tidal stripping from the SMC during its most recent encounter 100 - 300 Myr ago with the LMC. Tatton (2018) and Tatton et al. (2020) showed how the luminosity function of the RC varies across all VMC tiles belonging to the SMC, while Omkumar et al. (2021) have investigated the RC bi-modality using Gaia DR2 data. Additional evidence supporting the tidal stripping of SMC material during the most recent encounter comes in the form of accreted red giant branch stars belonging to the SMC and discovered in the LMC (Olsen et al. 2011), while Dobbie et al. (2014) found signatures of tidally stripped red giant branch stars in the outskirts of the SMC.

In this paper, we present a detailed analysis of the spatial distribution of different stellar populations in the periphery of the MCs using data from the VISTA Hemisphere Survey (VHS; McMahon et al. 2013). VHS enables us to obtain a comprehensive view of the morphology in the outskirts of the MCs over a continuous area, allowing us to confirm the presence of known substructures while also finding new ones. Furthermore, we also investigate the properties of the double RC feature, beyond the main body of the SMC, using a combination of VHS and VMC data. Identifying stellar substructures around the MCs using morphology and luminosity function studies is very important to understand the nature of the interactions between the MCs and will provide inputs for theoretical models. The paper is organised as follows, Section 2 describes the data set used in our study and our selection criteria, Section 3 focuses on the outer morphology of the MCs, while Section 4 studies the properties of the double RC feature. Finally, Section 5 presents a discussion and summary of our results.

\section{OBSERVATIONS AND DATA SELECTION}

\subsection{Data}

We used data from VHS, a near-infrared (NIR) survey, that covers the whole southern hemisphere when combined with other firstgeneration VISTA Public Surveys. VHS is carried out by the $4.1 \mathrm{~m}$ Visible and Infrared Survey Telescope for Astronomy (VISTA; Sutherland, Will et al. 2015) and the VISTA infrared camera (VIRCAM; Dalton et al. 2006; Emerson et al. 2006). It aims to cover $18,000 \mathrm{deg}^{2}$ with observations in at least two photometric bands, $J$ and $K_{\mathrm{S}}$ (central wavelengths: $1.25 \mu \mathrm{m}$ and $2.15 \mu \mathrm{m}$, respectively). Median $5 \sigma$ point source limits of $J=19.3 \mathrm{mag}$ and $K_{\mathrm{S}}=18.5 \mathrm{mag}$ (in the Vega system) are achieved with a minimum exposure time of $60 \mathrm{~s}$ per waveband. VHS includes three programmes: VHS-GPS (Galactic Plane Survey), covering a region of $\approx 8200 \mathrm{deg}^{2}$ with exposure times of $60 \mathrm{~s}$ in the $J$ and $K_{\mathrm{S}}$ bands; VHS-DES, covering a region of $\approx 4500 \mathrm{deg}^{2}$ with exposure times of 120 s in the $J H K_{\mathrm{S}}$ 




Figure 1. Schematic figure showcasing the footprints of the VHS and VMC surveys. The borders of the VHS sub-surveys are indicated in red and those of observational gaps in black, while the area covered by VMC tiles is displayed in light grey. The projection origin for the maps is set at $\left(\mathrm{RA}_{0}\right.$, $\left.\operatorname{Dec}_{0}\right)=\left(81.24^{\circ},-69.73^{\circ}\right)$ corresponding to the densest point in the LMC bar (de Vaucouleurs \& Freeman 1972).

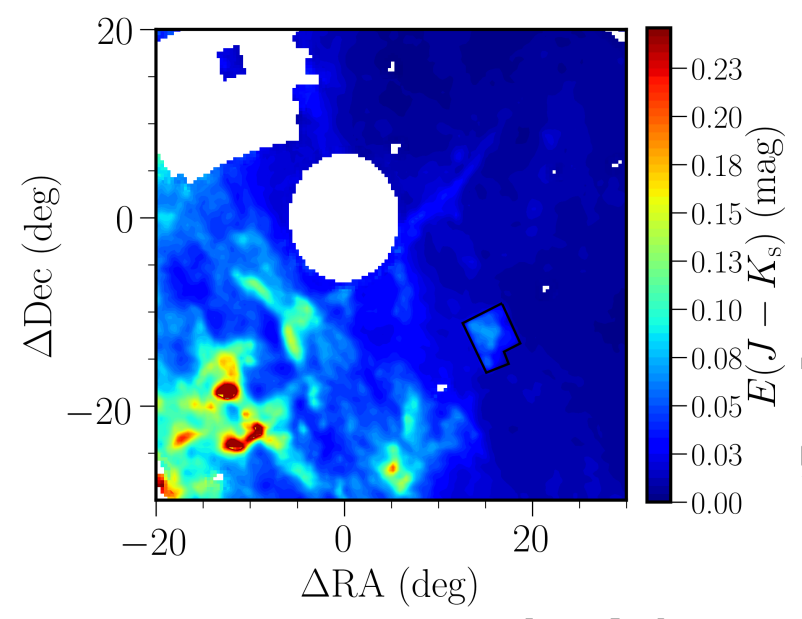

Figure 2. Distribution of reddening derived from the Schlegel et al. (1998) dust map as well as from the star formation history study of the main body of the SMC (outlined in black) by Rubele et al. (2018) across the footprint analysed in this paper. The map is centred at $\left(\mathrm{RA}_{0}, \mathrm{Dec}_{0}\right)=\left(81.24^{\circ}\right.$, $\left.-69.73^{\circ}\right)$ and the colour bar shows the yariation of the $E\left(J-K_{\mathrm{S}}\right)$ colour excess. White patches refer to the central regions occupied by the LMC and to the incomplete VHS observations towards the NE, as well as to a few tiles in the outskirts of the MCs.

bands and VHS-ATLAS, covering a region of $\approx 5000 \mathrm{deg}^{2}$ evenly divided between the North and South Galactic caps with exposure times of $60 \mathrm{~s}$ in the $Y J H K_{\mathrm{S}}$ bands. VHS data from all three programmes border the MCs (Figure 1). Our study makes use of all VHS observations obtained until 2017 September 30th.

We also make use of data from the VMC survey (Cioni et al. 2011), a deep NIR multi-epoch imaging survey of the MCs providing data in three photometric bands reaching $5 \sigma$ limits of $Y=21.9$,
$J=22$, and $K_{\mathrm{S}}=21.5 \mathrm{mag}$ (in the Vega system). VMC observed the LMC (105 deg $\left.{ }^{2}\right)$, SMC (42 deg $\left.{ }^{2}\right)$, the Magellanic Bridge (21 deg $\left.{ }^{2}\right)$ and the Magellanic Stream $\left(3 \mathrm{deg}^{2}\right)$. In this study we make use of observations in the SMC and Bridge components (Figure 1). VHS data is combined with VMC data in order to fill observational gaps in the Magellanic Bridge (two small areas within it, see Figure 1) and to study the luminosity function of RC stars with continuous coverage within $10 \mathrm{deg}$ from the centre of the SMC. Our selection criteria for VMC sources are the same as those used for VHS sources (see below). Duplicate sources that are present in both surveys (e.g. in the outskirts of the LMC and SMC, as well as the Magellanic Bridge) were eliminated by keeping VMC detections.

The VISTA Data Flow System (VDFS; Irwin et al. 2004) was used for data reduction while the data were extracted from the VISTA Science archive (VSA ${ }^{1}$; Cross et al. 2012). The VHS data allow us to explore resolved stellar populations in the periphery of the MCs and to detect sources just below the RC. VHS showcases an improvement in depth over previous NIR surveys such as the Two Micron All Sky Survey (2MASS; Skrutskie et al. 2006) and the DEep Near Infrared Survey of the Southern Sky (DENIS; Cioni et al. 2000), which have limiting magnitudes $\approx 4$ mag brighter in $K_{\mathrm{S}}$. VHS is the deepest NIR survey covering the entire southern hemisphere to date, and although it is by design much shallower than its optical counterparts such as Gaia DR2 and the DES (Abbott et al. 2018), VHS provides an essential contribution to the multi-wavelength view of the periphery of the MCs.

Figure 2 shows the line-of-sight Galactic dust reddening derived from the Schlegel et al. (1998) dust maps. In the Bar and Wing regions of the SMC, Schlegel et al. (1998) measurements can be unreliable due to contamination by SMC stars or unresolved temperature structure. We combined the Schlegel et al. (1998) reddening map with reddening derived from the star formation history study of the main body of the SMC by Rubele et al. (2018). Rubele et al. (2018) used VMC data across an area of $23.57 \mathrm{deg}^{2}$ with a bin size of $0.143 \mathrm{deg}^{2}$ and reconstructed the observed CMDs using stellar partial models, recovering the best-fitting extinction $A_{V}$ as an additional output of the star formation rate. The combination of the Schlegel et al. (1998) and Rubele et al. (2018) maps encompasses the entire area studied in this paper. For the Schlegel et al. (1998) map, $E(B-V)$ values were obtained using the python module SFDMAP ${ }^{2}$ and converted to $E\left(J-K_{\mathrm{S}}\right)$ values using coefficients from González-Fernández et al. (2018). These coefficients have been calculated assuming $R_{V}=3.1$ and account for the Schlafly \& Finkbeiner (2011) recalibration. For the Rubele et al. (2018) map, we converted $A_{V}$ to $E(B-V)$ values assuming $R_{V}=$ 3.1 and used the same coefficients to convert them to $E\left(J-K_{\mathrm{S}}\right)$ values. Despite the spatial distribution of reddening being generally low in the periphery of the MCs with $E\left(J-K_{\mathrm{S}}\right) \approx 0.10 \mathrm{mag}$, particularly at the location of the substructures traced in this study, filamentary dust features with high reddening values are present near the Galactic disc. Moreover, the sharp borders (owing to the VISTA field-of-view) suggest that the level of reddening in the immediate outskirts of the SMC may be underestimated as we only account for the foreground reddening and not the intrinsic reddening of the SMC. In the analysis which follows, we therefore correct for reddening. However, the central regions of the LMC and SMC, including the region covered by the Rubele et al. (2018) reddening 

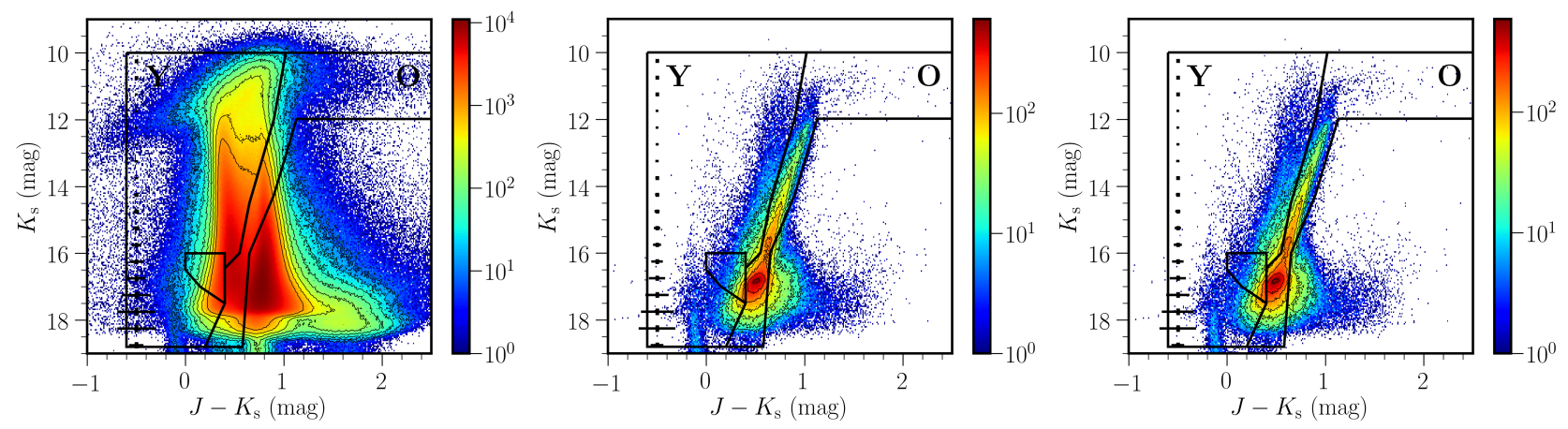

Figure 3. NIR $\left(J-K_{\mathrm{S}}, K_{\mathrm{s}}\right)$ Hess diagrams of the stellar periphery of the MCs before the cross-match with Gaia DR2 data (left), after the cross-match using the selection criteria to reduce the presence of MW stars (middle), and after correcting for reddening (right). The colour scale indicates the stellar density on a logarithmic scale whereas horizontal bars show the photometric uncertainties as a function of magnitude. Two different classes of objects are enclosed by black lines, $\mathrm{Y}$ referring to young stars and $\mathrm{O}$ referring to old stars. Stars belonging to the polygonal area $\left(16 \leq K_{\mathrm{S}} \leq 17.5 \mathrm{mag}\right)$ are not included in our study as they have mixed ages.

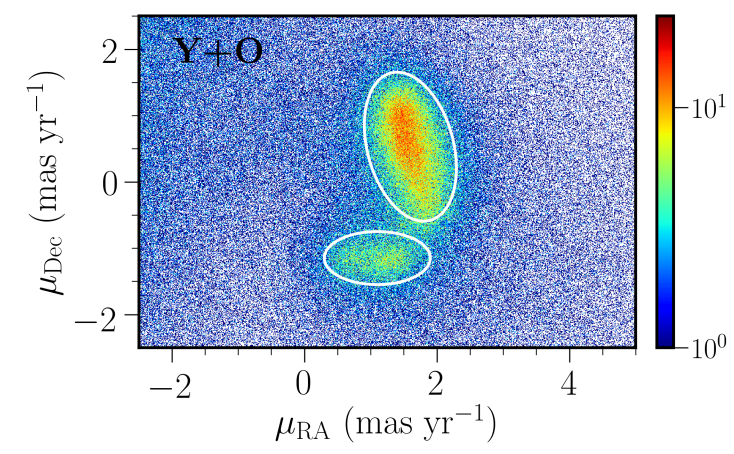

Figure 4. Stellar proper motions towards the direction of the MCs. Most of the objects with LMC and SMC proper motions are enclosed within the large and small ellipse, respectively, whereas many of the objects outside these ellipses belong to the MW.

map, have been omitted from the morphological maps to enhance the distribution of stars in the outer regions.

\subsection{Selection of young and old stellar populations}

The selection of different stellar populations was based on $\left(J-K_{\mathrm{S}}, K_{\mathrm{S}}\right)$ CMDs (see Figure 3$)$. We selected unique objects $($ priOrSec $\leqslant \theta$ or priOrSec $=$ frameSetID) from the vhssource table detected in both $J$ and $K_{\mathrm{s}}$ bands, classified as stars with at least a $70 \%$ probability ( $\mathrm{flag}$ mergedclass $=-1$ or mergedclass $=-2$ ) and with photometric errors less than 0.2 mag. The AperMag3, which corresponds to the default point source aperture corrected magnitude ( $2^{\prime \prime}$ aperture diameter), was retrieved for each source in each waveband. No selection criteria based on extraction quality flags ( $f l a g$ ppErrbits) were applied. VISTA magnitudes were transformed to the Vega system by adding $0.011 \mathrm{mag}$ to the $K_{\mathrm{s}}$ band while no corrections are required in the $J$ band (Gonźález-Fernández et al. 2018).

Stars belonging to the MCs were divided into two regions: region $Y$ occupied by young stars, including tip of the main sequence and supergiant stars, and region $\mathrm{O}$ occupied by old stars, including red giant branch, asymptotic giant branch, and RC stars. These two regions result from the combination of CMD regions established by El Youssoufi et al. (2019): region Y comprises regions A, B, C, $\mathrm{I}, \mathrm{G}, \mathrm{H}$ and region $\mathrm{O}$ includes regions $\mathrm{D}, \mathrm{E}, \mathrm{J}, \mathrm{K}, \mathrm{M}$. Region $\mathrm{Y}$ has been extended to $K_{\mathrm{S}}=10 \mathrm{mag}$, instead of $K_{\mathrm{S}}=11.98 \mathrm{mag}$ and to $J-K_{\mathrm{S}}=-0.6 \mathrm{mag}$ instead of $J-K_{\mathrm{S}}=-0.2 \mathrm{mag}$, to maximise the number of stars after their cross-correlation with Gaia DR2. Both regions $\mathrm{Y}$ and $\mathrm{O}$ have been limited to $K_{\mathrm{s}}=18.8 \mathrm{mag}$ instead of $K_{\mathrm{S}}=19.8 \mathrm{mag}$, to reflect the sensitivity of the VHS observations. Due to the small number of stars in the periphery of the MCs, we combined several regions as opposed to using them separately. Studies of MC objects are prone to both foreground and background contamination. Most background galaxies were omitted from our selection by adopting the object classification flag mergedclass and avoiding the reddest region of the CMD $\left(J-K_{\mathrm{S}}>1\right.$ mag and $K_{\mathrm{S}}>13 \mathrm{mag}$ ). However, this region may contain reddened MCs sources and a minority of red giant branch stars scattered from the adjacent region of old stars. To reduce the presence of MW stars in our sample, we combined data from Gaia DR2 with VHS and VMC data $^{3}$, retaining only sources with a maximum cross-matching distance of 1 " and parallaxes $\leq 0.2$ mas.

In addition, we applied a proper motion selection. We examined the stellar density of objects belonging to regions $\mathrm{Y}$ and $\mathrm{O}$ in proper motion space and determined two ellipses around the LMC and SMC proper motion distributions (Figure 4). Throughout the paper, we refer to $\mu_{\mathrm{RA}} \cos (\mathrm{Dec})$ as $\mu_{\mathrm{RA}}$. The LMC ellipse is centred at $\mu_{\mathrm{RA}}=1.60 \mathrm{mas} \mathrm{yr}^{-1}, \mu_{\mathrm{Dec}}=0.53 \mathrm{mas} \mathrm{yr}^{-1}$, has a semi-major axis of $1.35 \mathrm{mas} \mathrm{yr}^{-1}$, a semi-minor axis of $0.85 \mathrm{mas} \mathrm{yr}^{-1}$, and is rotated by $15^{\circ}$, whereas the SMC ellipse is centred at $\mu_{\mathrm{RA}}=1.10{\mathrm{mas} \mathrm{yr}^{-1}}^{-1}$

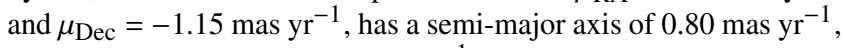
a semi-minor axis of 0.40 mas $^{-1} r^{-1}$ and no rotation. Objects belonging to these two ellipses constitute our morphology dataset which contains 312,748 sources, encompassing an area of 1800 $\mathrm{deg}^{2}$ around the MCs.

In order to assess the detection limit of the VHS survey, we examined the normalised number of sources in each sub-survey as a function of magnitude compared with the 2MASS and VMC surveys. We used circular areas of $0.5^{\circ}$ radius with the following (RA, Dec) centres: 2 MASS $\left(32.9^{\circ},-75.0^{\circ}\right)$, VMC $\left(32.9^{\circ},-75.0^{\circ}\right)$, VHS-ATLAS $\left(43.5^{\circ},-77.8^{\circ}\right)$, VHS-GPS $\left(111.50^{\circ},-75.66^{\circ}\right)$, and VHS-DES $\left(42.00^{\circ},-60.97^{\circ}\right)$. We found that the VHS programmes ATLAS, GPS, and DES have detection limits in $J$ of 19.84 mag, $19.55 \mathrm{mag}$ and $20.36 \mathrm{mag}$, respectively, while in $K_{\mathrm{S}}$ the detection

\footnotetext{
3 The catalogues have been cross-matched by G. Matijevič using his own software tools.
} 

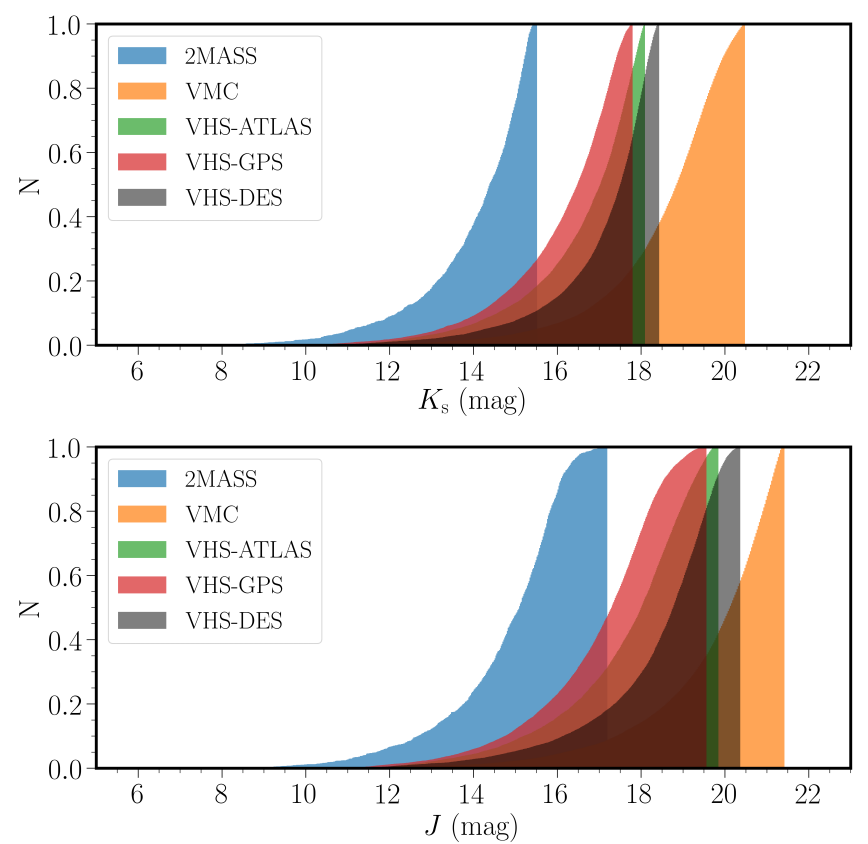

Figure 5. Comparison between the detection limits of 2MASS, VMC, VHSGPS, VHS-DES, and VHS-ATLAS in circular areas of $0.5^{\circ}$ radius in the $J$ (left) and $K_{\mathrm{S}}$ (right) bands. The number of sources is normalised.

limits are of $18.08 \mathrm{mag}, 17.79 \mathrm{mag}$ and $18.42 \mathrm{mag}$, respectively. Only sources detected in both $J$ and $K_{\mathrm{S}}$ with photometric uncertainties less than 0.2 mag were included. These values show that stellar populations brighter than and including the bulk of the RC are not significantly affected by incompleteness.

\subsection{Selection of RC stars around the SMC}

To study the distribution of RC stars in the outskirts of the SMC we proceeded as follows. We combined VHS with VMC observations of the SMC and Bridge which are within $10^{\circ}$ from the SMC's optical centre. Due to the smaller number of stars in the periphery of the SMC than in the main body of the galaxy, we divided the SMC into circular annuli of radii $1^{\circ}, 2^{\circ}, 3^{\circ}, 4^{\circ}, 5^{\circ}, 6^{\circ}, 7^{\circ}, 8^{\circ}$ and $10^{\circ}$ from the centre of the galaxy and each annulus into four regions North $(\mathrm{N})$, East (E), South (S) and West (W), see Figure 6 (left). In the $\left(J-K_{\mathrm{S}}\right.$, $K_{\mathrm{S}}$ ) CMDs, specific stellar populations are indicated (Figure 6 right). Foreground MW stars were removed following a similar procedure as that described above using a combination of parallax and proper motion cuts. For the proper motion selection, we examined the stellar density in proper motion space of sources up to $10^{\circ}$ from the centre of the SMC and defined an ellipse with the following parameters:

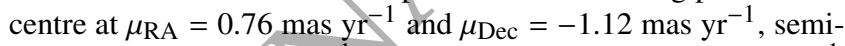

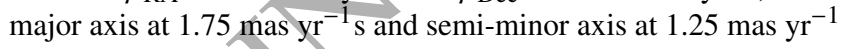
(Figure 7). This ellipse is larger than the one defined in Section 2.2, to distinguish between LMC and SMC stars, in order to include more RC stars in the outer regions of the SMC (not necessarily associated to the SMC itself) and at the same time minimise the influence of the MW. The small difference between the centres of the two proper motion SMC ellipses reflects the distribution of the type of stellar population dominating the inner and outer regions of the SMC. Zivick et al. (2020) showed that the centre of the proper motion distribution of red giant stars is clearly offset from where one would place the centre of the distribution of main sequence stars. The central region of the SMC is masked out in Figure 4 but it is included in Figure 7 . In the $\left(J-K_{\mathrm{S}}, K_{\mathrm{S}}\right)$ CMDs, we defined a box to encompass the full extent of RC stars, take into account the influence of RGB stars and minimise contamination from neighbouring stellar populations (Sect. 4 and Fig. 8). The bright limit $\left(K_{\mathrm{S}}=15 \mathrm{mag}\right)$ was chosen to include stars beyond the RGB bump for better modelling of the RGB population, while the faint limit $\left(K_{\mathrm{S}}=18.5 \mathrm{mag}\right)$ represents the $5 \sigma$ point source limit in the $K_{\mathrm{S}}$ band. On the red side, neighbouring populations are RGB and MW stars. Therefore, the red borders of the box are defined as those of K (RGB) and F (MW) from El Youssoufi et al. (2019). On the blue side, the main contaminants are horizontal branch stars, supergiant and subgiant stars. Beyond a radius of $4^{\circ}$, photometric. uncertainties and metallicity variations elongate the distribution of RC stars, shifting also RGB stars to bluer colours. Therefore, the blue limit for $K_{\mathrm{S}} \leq 16.5 \mathrm{mag}$ is the same as that of region I (red supergiants) from El Youssoufi et al. (2019) to include the full width of the RGB, while for $K_{\mathrm{S}} \geq 16.5 \mathrm{mag}$, the limit is shifted by $\sigma_{J-K_{\mathrm{S}}}=0.13$ mag to include RC stars. RR Lyrae stars are generally confined to a compact region below the RC $\left(0 \leq J-K_{\mathrm{S}} \leq 0.5 \mathrm{mag}\right.$, $\left.17.7 \leq K_{\mathrm{S}} \leq 19.4 \mathrm{mag}\right)$. Therefore, they mostly affect the fainter end of the RGB.

\section{STELLAR PERIPHERY OF THE MAGELLANIC CLOUDS}

We used the NIR $\left(J-K_{\mathrm{S}}, K_{\mathrm{S}}\right)$ CMD to divide the stellar populations into young and old stars. The VHS photometry reaches one magnitude below the RC. Figure 9 outlines the morphological features discussed in this work, while Figure 10 shows morphology maps of the spatial density of young, old and young+old stars using LMC, SMC and LMC or SMC proper motion selections (Section 2.2). The bin size is $0.12 \mathrm{deg}^{2}$. The projection origin for the maps is set at $\left(\mathrm{RA}_{0}, \mathrm{Dec}_{0}\right)=\left(81.24^{\circ},-69.73^{\circ}\right)$ corresponding to the densest point in the LMC bar (de Vaucouleurs \& Freeman 1972). The transformation of coordinates from angular to Cartesian was performed through a zenithal equidistant projection (van der Marel 2001). The LMC globular clusters NGC $1841\left(\Delta \mathrm{RA}=1^{\circ}, \Delta \mathrm{Dec}=-14^{\circ}\right)$, Reticulum $\left(\triangle \mathrm{RA}=6^{\circ}, \Delta \mathrm{Dec}=10^{\circ}\right)$ and the MW globular cluster NGC 1261 $\left(\triangle \mathrm{RA}=17.5^{\circ}, \Delta \mathrm{Dec}=10^{\circ}\right)$ appear as clear overdensities in the morphology maps.

\subsection{Morphological features}

The morphology maps reveal striking substructures in the periphery of the MCs. The Northern Substructure 1 of the LMC detected by Mackey et al. (2016) $\left(\Delta \mathrm{RA}=0^{\circ}, \Delta \mathrm{Dec}=15^{\circ}\right)$ is most prominent in the old population extending to $\sim 20^{\circ}$ from the LMC centre, it is present also in the map of the young population but to a lesser radial extent. The extension of this feature towards the NE is not visible due to incomplete VHS observations. We also detect two substructures on the eastern side of the LMC (Eastern Substructures 1 and 2), revealed at $\left(\Delta \mathrm{RA}=-15^{\circ}, \Delta \mathrm{Dec}=1^{\circ}\right)$ and $\left(\Delta \mathrm{RA}=-15^{\circ}\right.$, $\left.\Delta \mathrm{Dec}=-6^{\circ}\right)$, and emerging in the direction of the Galactic disc. We find that these substructures are present despite the strict proper motion cuts we applied (see Section 2.3), suggesting they belong to the LMC rather than to the MW. In the young population and somewhat closer to the LMC disc, Eastern Substructure 1 delineates an approximate circle while Eastern Substructure 2 is similar to a thin stream. There is also a high-density clump between the two substructures at about $\left(\Delta \mathrm{RA}=-10^{\circ}, \Delta \mathrm{Dec}=0^{\circ}\right)$. These features 



Figure 6. (left) Spatial distribution of stars around the SMC and plotted annuli from $0^{\circ}$ to $10^{\circ}$ further divided into 4 regions: N, W, S, and E. VMC tiles are also shown. (right) NIR $\left(J-K_{\mathrm{S}}, K_{\mathrm{s}}\right)$ Hess diagram (corrected for reddening) of all stars in the region shown in the right panel. Specific stellar populations are indicated as follows: asymptotic giant branch (AGB) stars, red supergiant (RSG) stars, tip of main sequence (MS) stars, intermediate-age Helium burning (Int. $\mathrm{HeB}$ ) stars as well as RGB, RC and MW stars while RGBb refers to the RGB bump.

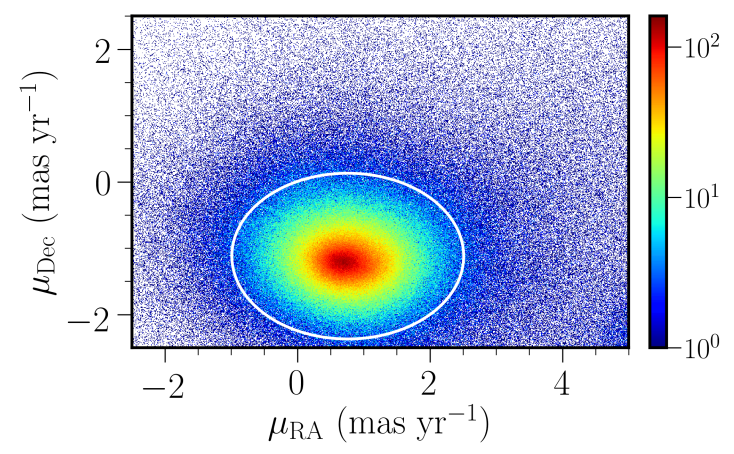

Figure 7. Stellar density of objects up to $10^{\circ}$ from the SMC centre in proper motion space. The ellipse encloses a region with a reduced number of MW stars.

are less prominent in the old population. de Vaucouleurs (1955) identified a circular feature in the eastern LMC disc at about $(\triangle \mathrm{RA}$ $=-14^{\circ}, \Delta \mathrm{Dec}=2^{\circ}$ ) which most likely coincides with Eastern Substructure 1. Eastern Substructure 2 is a new discoyery.

South of the LMC we find significant overdensities encompassing the locations of substructures previously identified by Mackey et al. (2018): Southern Substructure 1 at $\left(\Delta \mathrm{RA}=-2^{\circ}\right.$, $\left.\Delta \mathrm{Dec}=-12.5^{\circ}\right)$ and Southern Substructure 2 at $\left(\Delta \mathrm{RA}=6^{\circ}, \Delta \mathrm{Dec}\right.$ $=-10^{\circ}$ ). These substructures are most prominent in the old population. The LMC globular eluster NGC 1841 appears embedded in a low density substructure extending farther south than Substructures 1 and 2. This southernmost extension (Southern Substructure 3; $\left.\Delta \mathrm{RA}=2^{\circ}, \Delta \mathrm{Dec}=-17.5^{\circ}\right)$ probably corresponds to the stream-like feature previously detected by Belokurov \& Erkal (2019). In our maps it hás a high density in the old populations and its connection to Southern Substructure 1 is unclear. Southern Substructures 1 and 3 characterise the maps obtained from LMC-based proper motions, whereas Southern Substructure 2 is also present in SMC-based proper motion maps, suggesting a possible connection between the LMC and the SMC. This connection, which is supported by an overall extension of the SMC populations towards the east, may be associated with the Old Bridge (Belokurov et al. 2017).
The east of the SMC shows several protuberances that emerge from its more regular elliptical body. We confirm the SMC East substructure (Mackey et al. 2018) at $\left(\triangle \mathrm{RA}=18^{\circ}, \Delta \mathrm{Dec}=-14^{\circ}\right)$ and the extension of the Wing towards the Magellanic Bridge at $(\triangle \mathrm{RA}$ $\left.=9.5^{\circ}, \Delta \mathrm{Dec}=-6 \cdot 5^{\circ}\right)$. The SMC East substructure together with the SMCNOD overdensity (Pieres et al. 2017) west of the SMC at $\left(\triangle \mathrm{RA}=23^{\circ}, \Delta \mathrm{Dec}=-10^{\circ}\right)$ are most obvious in the old population, whereas the extension towards the Magellanic Bridge is better delineated in the young population. Between the Wing and SMCNOD features there is a clear overdensity extending outwards of the galaxy at $\left(\triangle \mathrm{RA}=19.5^{\circ}, \Delta \mathrm{Dec}=-7.5^{\circ}\right)$ which is most prominent in the old population. This feature, which we refer to as Northern Substructure 2, appears also in previous studies (e.g. Belokurov \& Erkal 2019), but it was not specifically mentioned. All of these SMC features are most prominent in maps created using SMC-based proper motions. Combining young and old populations enhances the level of substructure in the outskirts of the MCs with respect to the MW.

\subsection{Distance and proper motion of morphological features}

We determined distances to the morphological features by examining the luminosity function of RC stars within the areas defined in Figure 9 and using the CMD box outlined in Section 4. We employed a multi-component non-linear least squares fitting technique to characterise the peak of the magnitude distribution produced by RC stars for each feature. Figure (11) and Table 1 show the histograms and parameters, respectively, obtained for each morphological feature following the same methodology to study the double RC feature across the SMC (Section 4). The stellar populations seen in Eastern substructures 1 and 2 features lie at distances similar to that of the LMC ( $\sim 50 \mathrm{kpc}$, e.g. de Grijs \& Bono 2014b). This strengthens the hypotheses that the two substructures are either owing to material being tidally stripped from the disc, or are overdensities in the structure of the disc itself, ruling out that they may be shreds of a dwarf galaxy. We found that Southern Substructures 1 and 2, as well as the SMC East Substructure, are located at distances similar to that of the Magellanic Bridge. If Southern Substructure 2 and SMC East were to be associated to an Old Bridge then this structure would be 
at the same distance of the Magellanic Bridge. We also found that Southern Substructure 3 is located somewhat closer than the other southern substructures, but this is only marginal in view of the uncertainties involved and the difficult RGB subtraction compared to the other regions. Northern Substructure 1 appears instead further away than the LMC main body suggesting it is made of material tidally stripped from the LMC disc as a result of an interaction with the SMC (Mackey et al. 2016). On the other hand, Northern Substructure 2 appears at the same distance of the SMC main body and it is probably a feature of its ellipsoidal structure. The feature might be undergoing tidal stripping as a consequence of ram pressure effects which dislodged material from the galaxy (e.g. Tatton et al. 2020). Additionally, we find that the SMCNOD substructure is the most distant feature. SMCNOD is located $8^{\circ}$ away from the SMC centre and its stellar population is similar to that of the SMC main body which, in agreement with Pieres et al. (2017), suggests that it was probably removed from the galaxy.

We have also examined the proper motions of the stars enclosed within each morphological feature and compared them with the proper motion $\left(\mu_{\mathrm{RA}}, \mu_{\mathrm{Dec}}\right)$ of the $\operatorname{LMC}(1.871,0.391){\mathrm{mas} \mathrm{yr}^{-1}}^{-1}$ and SMC $(0.686,-1.237)$ mas $\mathrm{yr}^{-1}$ obtained using Gaia early DR3 (Gaia Collaboration et al. 2020). These values are consistent with the Gaia DR2 values derived by Gaia Collaboration et al. (2018) up to $6^{\circ}$ and $4^{\circ}$ from the LMC and SMC centres, respectively. Table 2 shows for each feature the number of stars $(\mathrm{Y}+\mathrm{O}$ according to the CMD selection described in Sect. 2.2), the angular distance from the LMC/SMC centres, and the median proper motions with the respective spread.

The features around the LMC are located at angular distances of $11-18^{\circ}$ from the centre, whereas those around the SMC are at $7-8^{\circ}$ from the centre. In RA the Southern and Northern substructures have higher proper motion values than the Easter substructures, all features show however proper motions lower than the LMC value. In Dec, Eastern substructures 1 and 2 as well as Southern Substructure 1 show higher proper motions than the LMC contrary to the other southern substructures and Northern Substructure 1 which show lower values. Assuming that the substructures belong to the LMC disc then the viewing perspective may account for proper motion

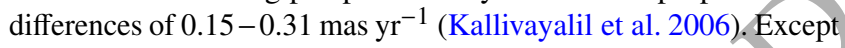
for Eastern Substructure 1 and 2, and for Southern Substructure 2 all other features have both proper motion components for which the difference with respect to the proper motion of the LMC can be explained by viewing perspective.

The proper motions of the features around the SMC appear higher in RA than in Dec compared to the values attributed to the galaxy. The structure of the SMC is complex and the influence of viewing perspective is difficult to estimate. The proper motion of the Magellanic Bridge is as expected the most discrepant (in RA) from the SMC because it connects both galaxies. Schmidt et al. (2020)

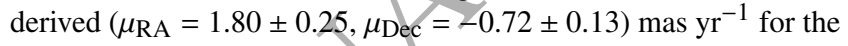
Bridge centre following a procedure that thoroughly removes the influence of MW stars. 

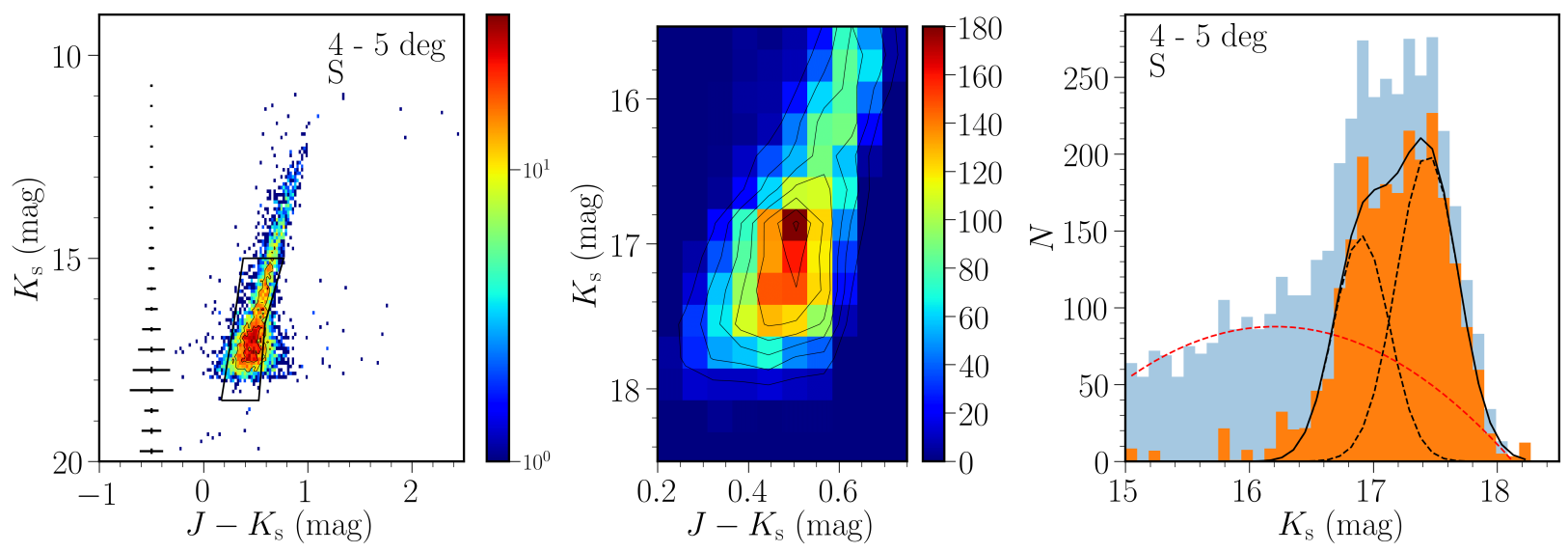

Figure 8. (left) Hess diagram (corrected for reddening) for SMC stars within the S sector and a $4-5^{\circ}$ annulus. (middle) Zoom-in on the selected RC region. (right) Luminosity function of RC stars in the same sector and annulus. The blue histogram shows the distribution of all stars while the orange histogram shows the distribution after the subtraction of the RGB component. The continuous line shows their total fit, while dashed lines represent the separate Gaussian components of the fits (black) and the quadratic polynomial term used to account for RGB stars (red).

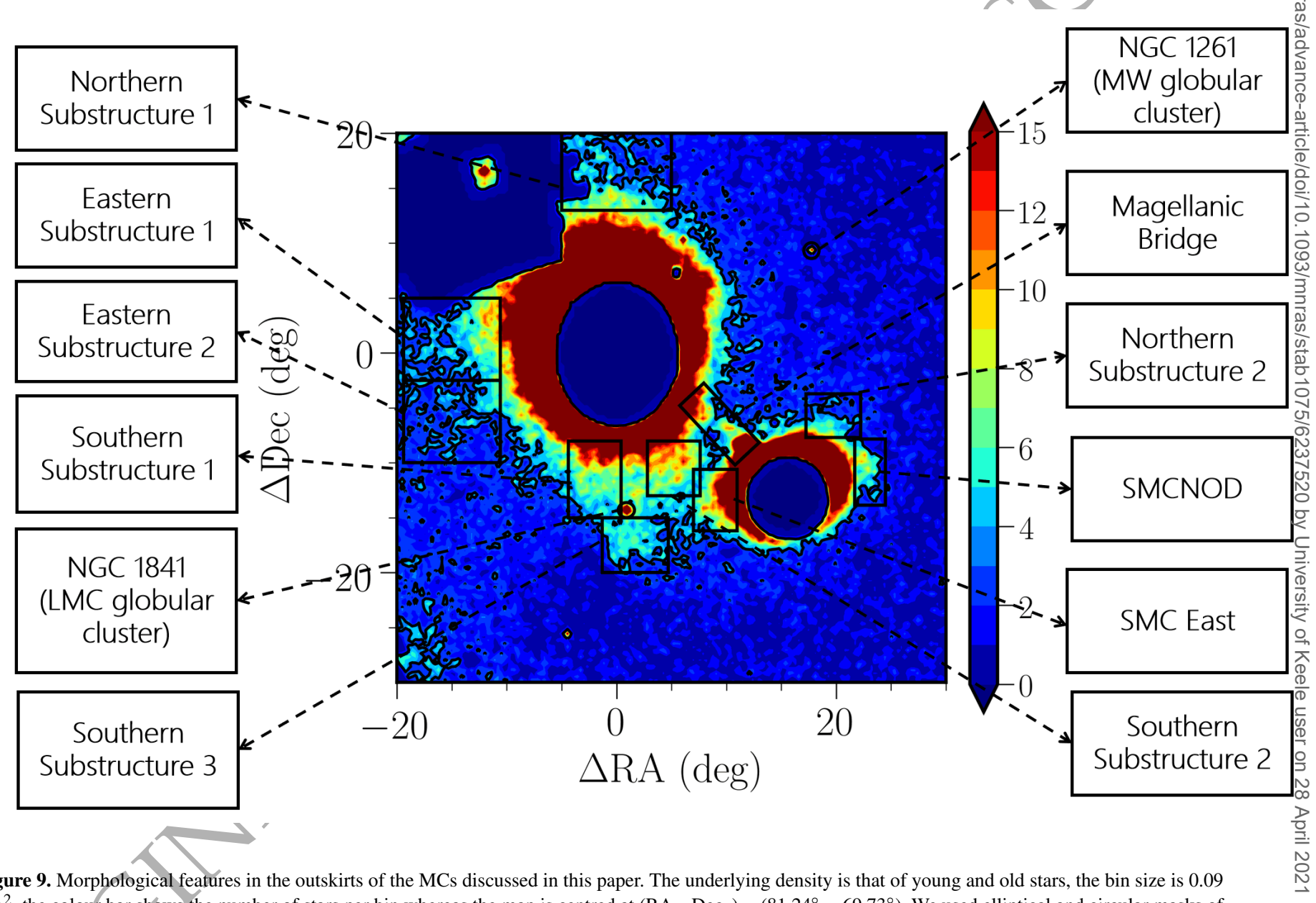

$\mathrm{deg}^{2}$, the colour bar shows the number of stars per bin whereas the map is centred at $\left(\mathrm{RA}_{0}, \operatorname{Dec}_{0}\right)=\left(81.24^{\circ},-69.73^{\circ}\right)$. We used elliptical and circular masks of the central region of the LMC and SMC, respectively, to enhance the distribution of stars in the outer regions. Some of these morphological features have already been reported in previous studies: the Eastern Substructure 1 by de Vaucouleurs (1955), the Northern Substructure 1 by Mackey et al. (2016), SMCNOD by Pieres et al. (2017), the Southern Substructure 3 by Belokurov \& Erkal (2019), the Southern Substructures 1 and 2 as well as the SMC East by Mackey et al. (2018), and the Magellanic Bridge by Hindman et al. (1963). The extension of the LMC and SMC outskirts outlined by the SMC East and Southern Substructure 2 embody the Old Bridge of Belokurov et al. (2017), whereas the Eastern Substructure 2 and Northern Substructure 2 have been highlighted for the first time in this work. 

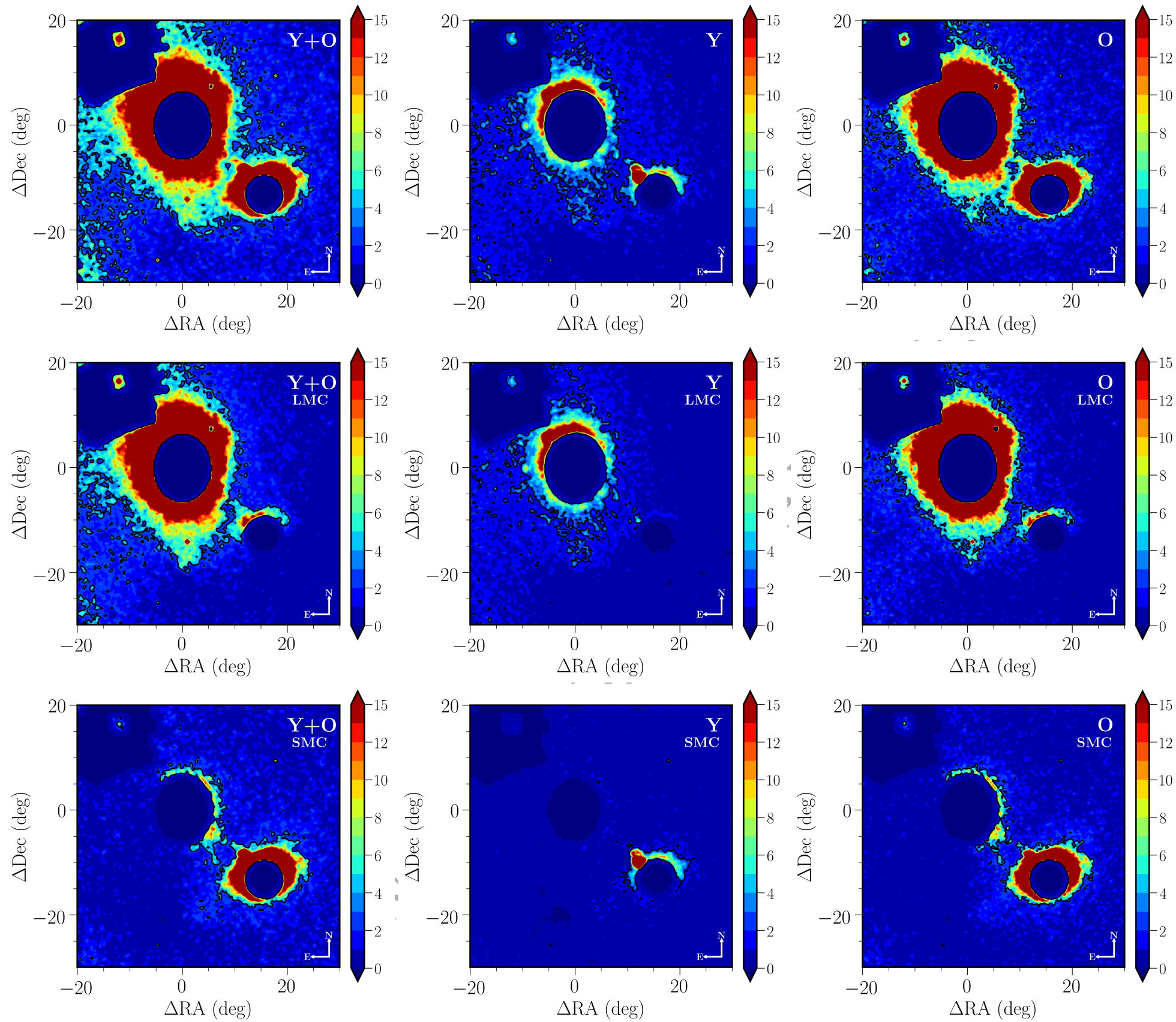

Figure 10. Morphology maps of young stars (Y; left) dominated by tip of MS and supergiant stars, old stars ( $\mathrm{O}$; middle) dominated RGB and RC stars, young and old stars ( $\mathrm{Y}+\mathrm{O}$; right) surrounding the MCs. The three rows show stars selected based on LMC or SMC proper motions (top), LMC proper motions (middle) and SMC proper motions (bottom). The bin size is $0.12 \mathrm{deg}^{2}$ and the maps are centred at $\left(\mathrm{RA}_{0}\right.$, Dec $\left.\mathrm{C}_{0}\right)=\left(81.24^{\circ}\right.$ $-69.73^{\circ}$ ). The colour bars show the numbers of stars per bin. The central regions of the LMC and SMC have been masked out to enhance the distribution of stars in the outer regions. 


\section{DOUBLE RED CLUMP FEATURE IN THE SMC}

Figure 6 (left panel) shows the spatial distribution of stars around the SMC, after applying the proper motion selection criteria (Figure 7 ), in sectors of annuli defined in Sect. 2. The main motivation behind using this cardinal division, is that the tail/Wing as well as the Magellanic Bridge are completely encompassed by the $\mathrm{E}$ sector (Fig. B3). Figure 6 (right panel) also shows a Hess diagram of the same stars outlining several stellar populations. A similar Hess diagram for stars within the $\mathrm{S}$ sector and the $4-5^{\circ}$ annulus is shown in Figure 8, whereas all Hess diagrams corresponding to all sectors in each annulus are shown in Appendix A (Figure A1). These CMDs show that the vertical extent of the RC population is more prominent in the eastern regions out to $10^{\circ}$ from the centre.

To study the RC stars in more detail, we constructed the luminosity function of the population, see Figure 8 (right panel) for stars within the $\mathrm{S}$ sector and the $4-5^{\circ}$ annulus and $\mathrm{A} 2$ for all sectors of each annulus, and obtained distances. Unlike Subramanian et al. (2017) and Tatton et al. (2020), we used the $J$ instead of the $Y$ band to its larger spatial coverage in the VHS survey. We constructed luminosity functions for RC stars using a bin size of 0.05 mag for regions with radius $R \leq 4^{\circ}$, and $0.1 \mathrm{mag}$ for $R \geq 4^{\circ}$ (the bin sizes are on the order of the errors in the $K_{\mathrm{S}}$ band). In order to evaluate the luminosity function, we employed a multi-component non-linear least squares fitting method. The CMD box, used to extract RC stars, also includes RGB stars, which we minimised by using a quadratic polynomial term. The main RC component is first modelled with one Gaussian and then, if the reduced $\chi^{2}$ parameter improves by more than 25 per cent when adding a second Gaussian, the second RC is deemed real. The parameters of the fits to the $\mathrm{RC}$ components and the reduced $\chi^{2}$ values are given in Table 3 , and the two-dimensional maps of the distances, means, and widths of the RC components are shown in Figure 14. Modelling the RGB component proved challenging for some regions $\left(5-6^{\circ} \mathrm{S}, 6-7^{\circ} \mathrm{S}, 7-8^{\circ}, 8-10^{\circ}\right)$, where only the brightest RGB component is present. This is owing to VHS observations being limited to just below the RC. In these cases, we modelled the RGB with a linear slope.

$K_{\mathrm{S}}$ is an excellent photometric band for absolute RC magnitude determination as it is less affected by reddening and systematic dependence on metallicity than optical bands. Many studies established median RC absolute magnitudes in the $K_{\mathrm{S}}$ band, which mostly agree on $M_{K}=-1.61 \mathrm{mag}$. Alves (2000) used $K$ magnitudes from the Caltech Two Micron Sky Survey (TMSS; Neugebauer \& Leighton 1969) of 238 Hipparcos RC stars and, under the assumption of no reddening, they found a value of $M_{K}=-1.61 \pm 0.03 \mathrm{mag}$. Using revised Hipparcos parallaxes Groenewegen (2008) finds $M_{K}=-1.54$ $\pm 0.04 \mathrm{mag}$, based on the 2MASS system. The difference in magnitude between the two studies was attributed to a selection bias due to a lack of data for bright nearby $\mathrm{RC}$ stars. This was confirmed by Laney et al. (2012) who found $M_{K}=-1.613 \pm 0.015 \mathrm{mag}$ from a sample of 226 bright RC stars using accurate $K$ band magnitudes. The drawback of the Alves (2000) study was the quality of the available IR photometry. More recent studies such as Hawkins et al. (2017) and Ruiz-Dern et al. (2018) favour the brighter magnitude of $M_{K}=-1.61 \mathrm{mag}$.

We converted the extinction corrected $K_{\mathrm{S}}$ magnitudes of the bright and faint RC components $\left(\mu_{0}\right.$ and $\left.\mu_{1}\right)$ to distance moduli, using the high-precision observations of RC stars in the solar neighbourhood by Laney et al. 2012. Their absolute magnitude in the 2MASS $K$ band corresponds to $M_{K}=-1.613 \pm 0.015 \mathrm{mag}$ whereas in the VISTA $K_{\mathrm{S}}$ band, it is $M_{K_{\mathrm{s}}}=-1.609 \pm 0.015 \mathrm{mag}$ (González-Fernández et al. 2018). However, since these stars are located in the solar neighbourhood, their absolute magnitude is expected to be different from those of RC stars in the SMC due to differences in metallicity, age and star formation rate. A correction for these population effects is needed and Salaris \& Girardi (2002) estimated it to be $-0.07 \mathrm{mag}$ in the $K_{\mathrm{S}}$ band. They obtained this term by simulating RC stars using stellar population models and including the star formation rate and age-metallicity relation derived from observations and then compared the difference in the absolute magnitudes in the SMC and solar neighbourhood to quantify the corresponding population effects. Once we obtained distance moduli for the different peaks, we calculated distance moduli uncertainties which include both the uncertainty on the absolute magnitude estimate as well as the peak magnitude uncertainty. We converted them to distances and distance uncertainties in kpc, and the values are given in Table 3. We followed the same methodology as Subramanian et al. (2017).

Most regions show a bi-modality in their luminosity function distribution, it was first discovered by Nidever ef al, (2013) and it can be due to several effects, including distance, line-of-sight depth as well population effects. Reddening has been ruled out as a significant contributor to the spread by Subramanian et al. 2017.

- $0^{\circ} \leq R \leq 2^{\circ}$ : The bi-modality is less pronounced here than in the outer regions. The difference in magnitude between the bright and faint RC components is on the order of $\sim 0.15 \mathrm{mag}$ and is generally less than the width of the bright Gaussian components. The difference between the two peaks is more pronounced in the southern regions. Note that these differences are ten times larger than the error on the peaks, but they are comparable with the width of the RC components. The width of the bright RC component is always larger than that of the faint component. The height of the faint component is always larger than the bright one, except for the northern and eastern regions in the $1^{\circ} \leq R \leq 2^{\circ}$ sector.

$2^{\circ} \leq R \leq 5^{\circ}$ : A clear separation between the bright and faint RC components becomes obvious in most regions of $2^{\circ} \leq R \leq 3^{\circ}$. The difference in magnitude becomes on the order of $\sim 0.4$ mag. Subramanian et al. (2017) found that this difference is too large to be attributed to any other effect but to different distances in the plane of the sky. The bi-modality has been detected by Nidever et al. (2013) at $4{ }^{\circ}$ and is likely due to material stripped from the SMC $200 \mathrm{Myr}$ ago. The bi-modality in the western regions was not seen by neither Nidever et al. (2013) or Subramanian et al. (2017).

- $5^{\circ} \leq R \leq 7^{\circ}$ : A bi-modality is only detected in a few regions. The dominant components are consistent with those in the inner SMC. In region $5-6^{\circ} \mathrm{W}$ we find a bright component, brighter than previously found, suggesting a foreground structure. In region $6-7^{\circ} \mathrm{N}$ we find a faint component, fainter than previously found which because of its large distance might belong to the structure known as the Counter Bridge (Diaz \& Bekki 2012), whereas a bright component in region 6-7 $\mathrm{S}$ might be linked to the Old Bridge (Belokurov et al. 2017). These three features might be influenced by a poor RGB subtraction.

- $7^{\circ} \leq R \leq 10^{\circ}$ : In the northern regions we detect both a bright and a faint component where the difference in magnitude between the two components is on the order of $\sim 0.7$. In the region $7-8^{\circ} \mathrm{S}$ we find a bright component as above, which is possibly linked to the Old Bridge and that at $8-10^{\circ}$ appears instead to the East. This demonstrates that the double $\mathrm{RC}$ feature is traceable out to $10^{\circ}$. A double component in the other regions is less well defined.

The fact that two components are present beyond the E sector at different radii demonstrates that the foreground structure in the form of a distance bi-modality is not solely owing to the Magellanic Bridge 

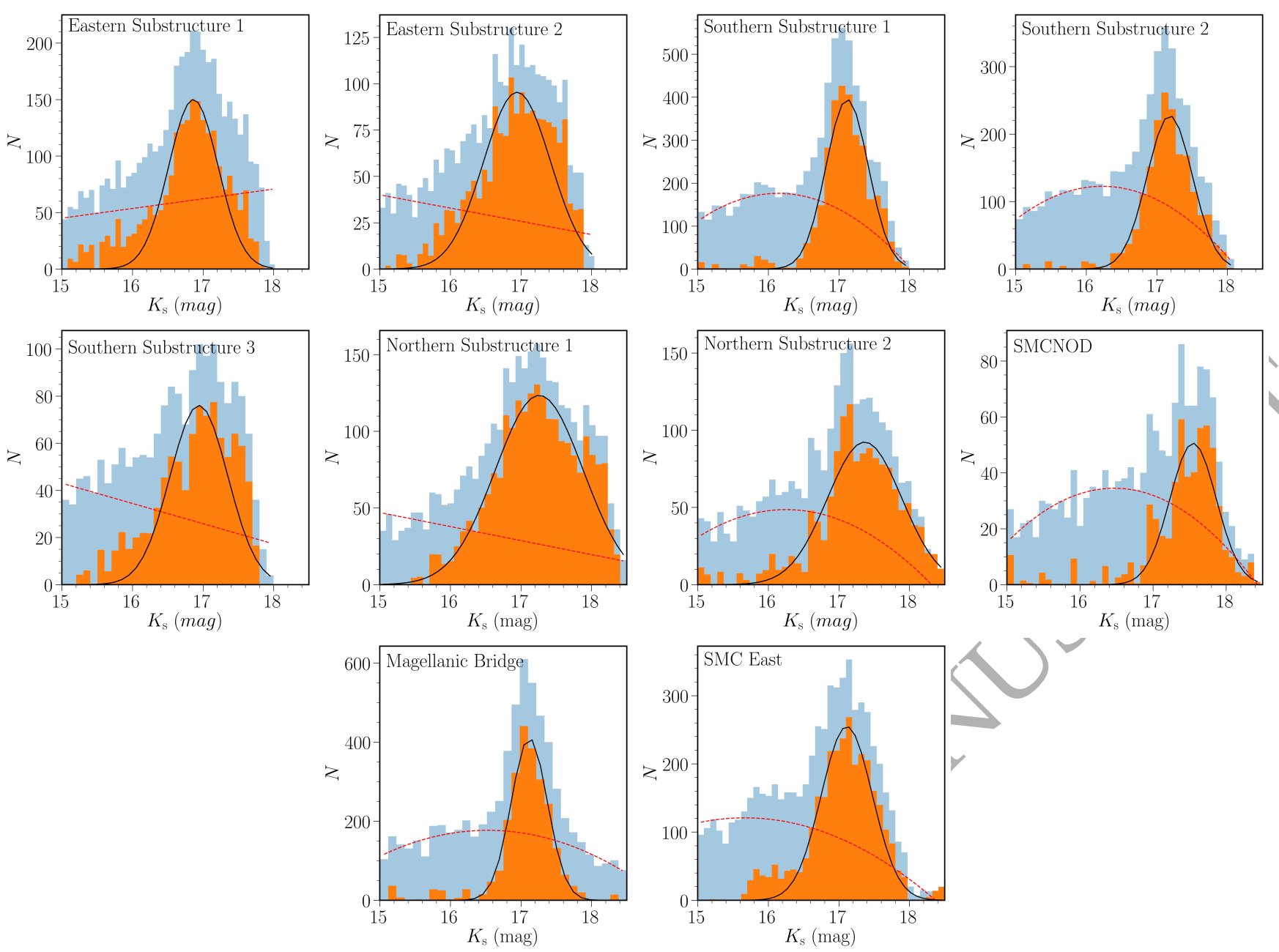

Figure 11. Luminosity function of the RC stars of the different morphological features outlined in Figure 9. The blue histograms show the observed luminosity functions, whereas orange histograms show the distributions after subtracting the RGB components, the continuous lines show the total fits to the distributions whereas the dashed lines represent the separate components of the fits. The bin size is of 0.09 mag.

Table 1. Gaussian parameters, distances and the reduced $\chi^{2}$ values of the profile fits to the luminosity function of the RC stars in different morphological features.

\begin{tabular}{lrrrrrr}
\hline Feature & $\begin{array}{r}\mu \\
(\mathrm{mag})\end{array}$ & $\begin{array}{r}\sigma \\
(\mathrm{mag})\end{array}$ & $\begin{array}{r}N \\
(\mathrm{counts})\end{array}$ & $\begin{array}{r}D \\
(\mathrm{kpc})\end{array}$ & $\begin{array}{r}\sigma_{\mathrm{D}} \\
(\mathrm{kpc})\end{array}$ & $\chi^{2}$ \\
\hline Eastern Substructure 1 & $16.86 \pm 0.08$ & 0.35 & $150 \pm 35$ & $47.9 \pm 1.9$ & 7.72 & 7.24 \\
Eastern Substructure 2 & $16.94 \pm 0.04$ & 0.47 & $95 \pm 8$ & $49.7 \pm 1.0$ & 10.76 & 4.85 \\
Southern Substructure 1 & $17.12 \pm 0.01$ & 0.29 & $395 \pm 19$ & $53.9 \pm 0.4$ & 7.21 & 2.94 \\
Southern Substructure 2 & $17.18 \pm 0.01$ & 0.32 & $227 \pm 10$ & $55.4 \pm 0.5$ & 8.18 & 1.30 \\
Southern Substructure 3 & $16.95 \pm 0.06$ & 0.40 & $531 \pm 10$ & $50.0 \pm 1.3$ & 9.20 & 5.95 \\
Northern Substructure 1 & $17.26 \pm 0.03$ & 0.62 & $123 \pm 6$ & $57.5 \pm 0.9$ & 16.44 & 2.68 \\
Northern Substructure 2 & $17.36 \pm 0.02$ & 0.52 & $92 \pm 5$ & $60.2 \pm 0.7$ & 14.44 & 1.71 \\
SMCNOD & $17.54 \pm 0.03$ & 0.31 & $51 \pm 5$ & $65.4 \pm 1.1$ & 9.36 & 1.51 \\
Magellanic Bridge & $17.12 \pm 0.01$ & 0.26 & $411 \pm 23$ & $53.9 \pm 0.5$ & 6.47 & 2.64 \\
SMC East & $17.11 \pm 0.03$ & 0.36 & $255 \pm 23$ & $53.7 \pm 0.8$ & 8.91 & 7.25 \\
\hline
\end{tabular}

and Wing. To further investigate the presence of the double RC feature at these different locations, we explored an alternative cardinal division where we used four sectors (NE, SE, NW, SW) that are $45^{\circ}$ offset counter-clockwise from the previous setup (N, E, S, W), see Appendix B. We performed the same analysis for the luminosity functions of the stars within these sectors and found comparable re- sults. This shows also that the faint component detected in the North, which might belong to the Counter Bridge, is present irrespective of the cardinal division scheme chosen. If the bi-modality in the luminosity function of RC stars is interpreted as a distance effect, it will correspond to a difference of $\sim 11 \mathrm{kpc}$ consistent with the findings of Subramanian et al. (2017) and Omkumar et al. (2021). 
Table 2. Proper motions of morphological features.

\begin{tabular}{lrrrrrrr}
\hline Feature & $\begin{array}{r}N \\
(\text { counts })\end{array}$ & $\begin{array}{r}\rho_{\mathrm{LMC}} \\
(\mathrm{deg})\end{array}$ & $\begin{array}{r}\rho_{\mathrm{SMC}} \\
(\mathrm{deg})\end{array}$ & $\begin{array}{r}\mu_{\mathrm{RA}} \\
\left(\mathrm{mas} \mathrm{yr}^{-1}\right)\end{array}$ & $\begin{array}{r}\sigma_{\mu_{\mathrm{RA}}} \\
\left(\mathrm{mas} \mathrm{yr}^{-1}\right)\end{array}$ & $\begin{array}{r}\mu_{\text {Dec }} \\
\left(\mathrm{mas} \mathrm{yr}^{-1}\right)\end{array}$ & $\begin{array}{r}\sigma_{\mu_{D e c}} \\
\left(\mathrm{mas} \mathrm{yr}^{-1}\right)\end{array}$ \\
\hline Eastern Substructure 1 & 4094 & 16 & - & 1.33 & 0.31 & 0.65 & 0.60 \\
Eastern Substructure 2 & 3622 & 17 & - & 1.31 & 0.33 & 0.49 & 0.57 \\
Southern Substructure 1 & 4270 & 13 & - & 1.61 & 0.33 & 0.59 & 0.47 \\
Southern Substructure 2 & 3040 & 12 & - & 1.66 & 0.33 & -0.12 & 0.43 \\
Southern Substructure 3 & 2286 & 18 & - & 1.52 & 0.34 & 0.13 & 0.65 \\
Northern Substructure 1 & 3460 & 16 & - & 1.58 & 0.31 & 0.09 & 0.53 \\
Northern Substructure 2 & 1720 & - & 7 & 1.20 & 0.27 & -1.05 & 0.20 \\
SMCNOD & 1116 & - & 7 & 0.93 & 0.29 & -1.13 & 0.15 \\
Magellanic Bridge & 9604 & 11 & 8 & 1.43 & 0.19 & -1.07 & 0.16 \\
SMC East & 5516 & - & 7 & 1.31 & 0.22 & -1.12 & 0.17 \\
\hline
\end{tabular}

The bright RC component which is detected throughout the N, S, and $\mathrm{E}$ sectors out to $10^{\circ}$ might be owing to a foreground substructure in the periphery of the SMC (including the Magellanic Bridge and the Old Bridge), whereas a faint component detected in the northern outermost regions might belong to the Counter Bridge. Whilst the substructures discussed in Sect. 3 are dominated by single components, the Northern Substructure 2 could also have two, in agreement with the analysis of the RC distribution across the galaxy.

\section{CONCLUSIONS}

In this paper, we have presented a study of the outer morphology of the MCs using NIR data from the VHS and $\left(J-K_{\mathrm{S}}, K_{\mathrm{S}}\right)$ CMDs to distinguish between young (tip of MS and supergiant stars) and old (RGB, AGB and RC stars) stellar populations. We minimise the influence of MW stars by using proper motion and parallax selection criteria from Gaia DR2. Our morphology maps demonstrate that it is possible to trace many of the diffuse stellar substructures present in the periphery of the MCs. Furthermore, we determine distances to these substructures using the luminosity function of RC stars. We confirm the detection of substructures discovered previously such as: the Northern and Southern features in the LMC disc by Mackey et al. (2016, 2018) and Belokurov \& Erkal (2019). We also characterise for the first time two new substructures: one on the eastern side of the LMC and one on the northern side of the SMC. A substructure on the east of the LMC was already present in de Vaucouleurs (1955) and it is possible that both eastern substructures are associated to the disc of the galaxy. The simulations described by Belokurov \& Erkal (2019) showed that the tidal influence of the MW and SMC are responsible for creating different substructures in the LMC. The SMC is responsible for creating the northern arm and a more prominent southern arm, while the MW's tidal field can also be responsible for creating, bending and extending the northern arm. The eastern substructures appear consistent with the influence of the MW deflecting the LMC disc. The substructure on the northern side of the SMC is probably associated to the ellipsoidal structure of the galaxy. Overall, we have been able to uncover a wealth of substructures in the periphery of the MCs, showcasing that their outskirts are susceptible to tidal interactions. Mapping these morphological features with fainter stellar populations and comparing their morphology to simulations can provide further constraints on the orbits of past encounters and the mass of the LMC.

We also present a study of the luminosity function of the RC stars from the centre of the SMC to a radius of $10^{\circ}$, using NIR data from the VHS and VMC surveys. Previous studies such as
Nidever et al. (2013), Subramanian et al. (2017), Tatton (2018) and Tatton et al. (2020) were limited to a radius of $4 \%$, therefore only covering the main body of the galaxy. Omkumar et al. (2021) used Gaia DR2 data to examine the luminosity function of RC stars up to $10^{\circ}$, however, they only detect a double red clump feature from $2.5^{\circ}$ to $5-6^{\circ}$ in the eastern regions. In this study we find that a bimodal $\mathrm{RC}$ feature is present in the eastern, northern and southern regions at different radii suggesting that tidal interactions have affected the entire inner eastern parts of the galaxy and not only its outskirts. In addition, our results reveal that RC stars form a background structure in the North of the SMC which might trace the Counter Bridge (Diaz \& Bekki 2012; Wang et al. 2019), and a foreground structure in the South which might trace the Old Bridge (Belokurov et al. 2017). This is consistent with previous studies based on metalpoor red giants (Dobbie et al. 2014), Cepheids and RR Lyrae stars (Ripepi et al. 2017, Jacyszyn-Dobrzeniecka et al. 2020a).

\section{ACKNOWLEDGEMENTS}

We are grateful to the anonymous referee who helped us shape the content and format of the paper with valuable suggestions. We thank the Cambridge Astronomy Survey Unit (CASU) and the Wide Field Astronomy Unit (WFAU) in Edinburgh for providing calibrated data products under the support of the Science and Technology Facility Council (STFC). This project has received funding from the European Research Council (ERC) under European Union's Horizon 2020 research and innovation programme (project INTERCLOUDS, grant agreement no. 682115). We thank Vasiliev Belokurov for the fruitful exchange concerning this work on multiple occasions. This research was supported by the DAAD with funds from the German Federal Ministry of Education and Research within an exchange grant between the Leibniz Institute of Astrophysics Potsdam (AIP, Germany) and the Indian Institute of Astrophysics (IIA, Bangalore). This study is based on observations obtained with VISTA at the Paranal Observatory under programmes 179.B-2003 and 179.A2010. This work has made use of data from the European Space Agency (ESA) mission Gaia (https: //www. cosmos. esa.int/ gaia), processed by the Gaia Data Processing and Analysis Consortium (DPAC, https://www. cosmos. esa. int/web/gaia/dpac/ consortium). Funding for the DPAC has been provided by national institutions, in particular the institutions participating in the Gaia Multilateral Agreement. SS acknowledges support from the Science and Engineering Research Board, India, through a Ramanujan Fellowship. This project has made extensive use of the Tool for OPerations on Catalogues And Tables (TOPCAT) software package 

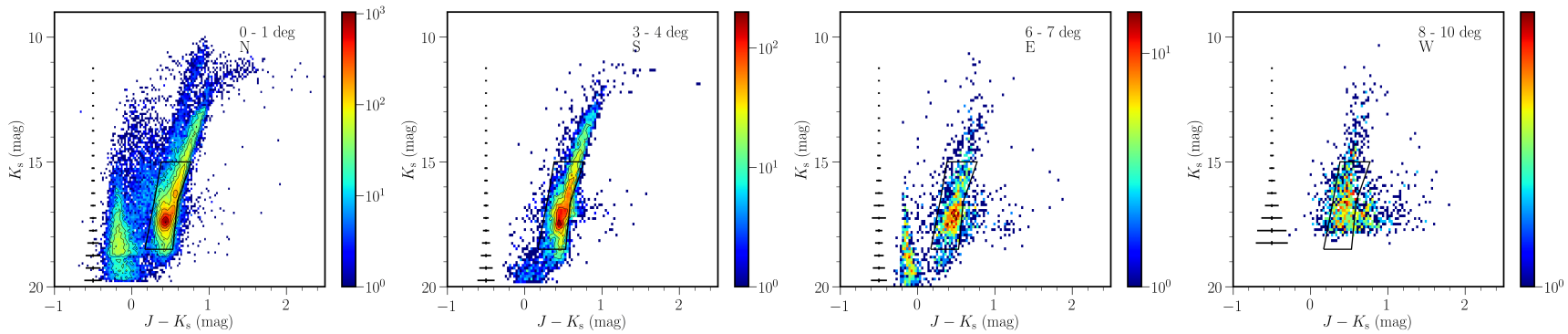

Figure 12. NIR $\left(J-K_{\mathrm{S}}, K_{\mathrm{S}}\right)$ Hess diagrams of a few of the SMC regions discussed in Sect. 4 and presented in full in Appendix A. The colour scale indicates the stellar density on a logarithmic scale per bin. The bin size is $0.027 \times 0.110 \mathrm{deg}^{2}$ and the black box limits the region defined to study the distribution of RC stars.
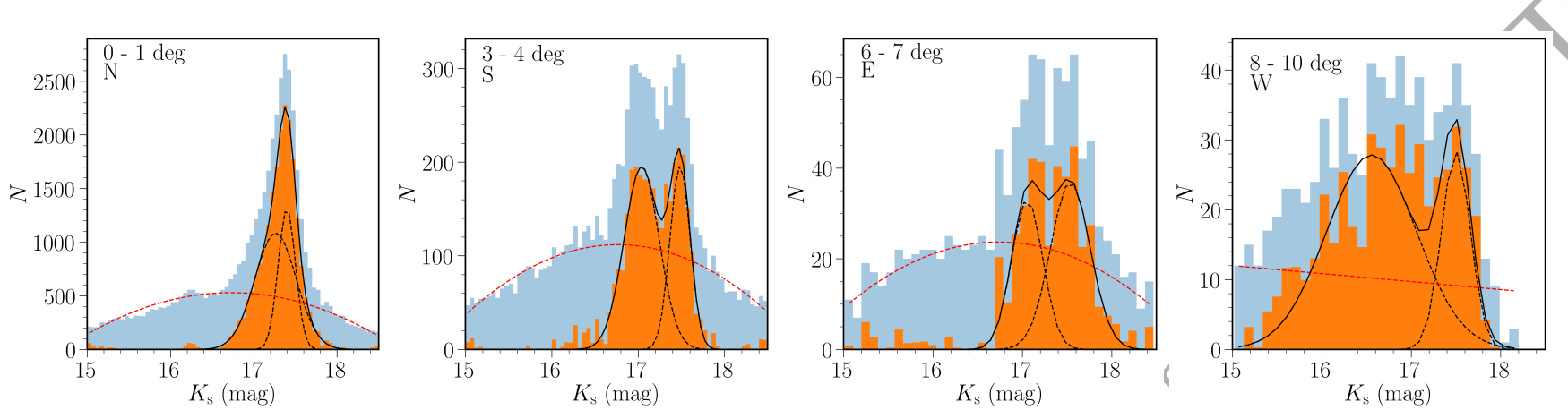

Figure 13. Luminosity function of the RC stars in a few different SMC regions discussed in Sect. 4 and presented in full in Appendix A. The blue histograms show the observed luminosity functions, whereas orange histograms show the distributions after subtracting the RGB components and the continuous lines show the total fits to these distributions whereas the dashed lines represent the separate components of the fits.
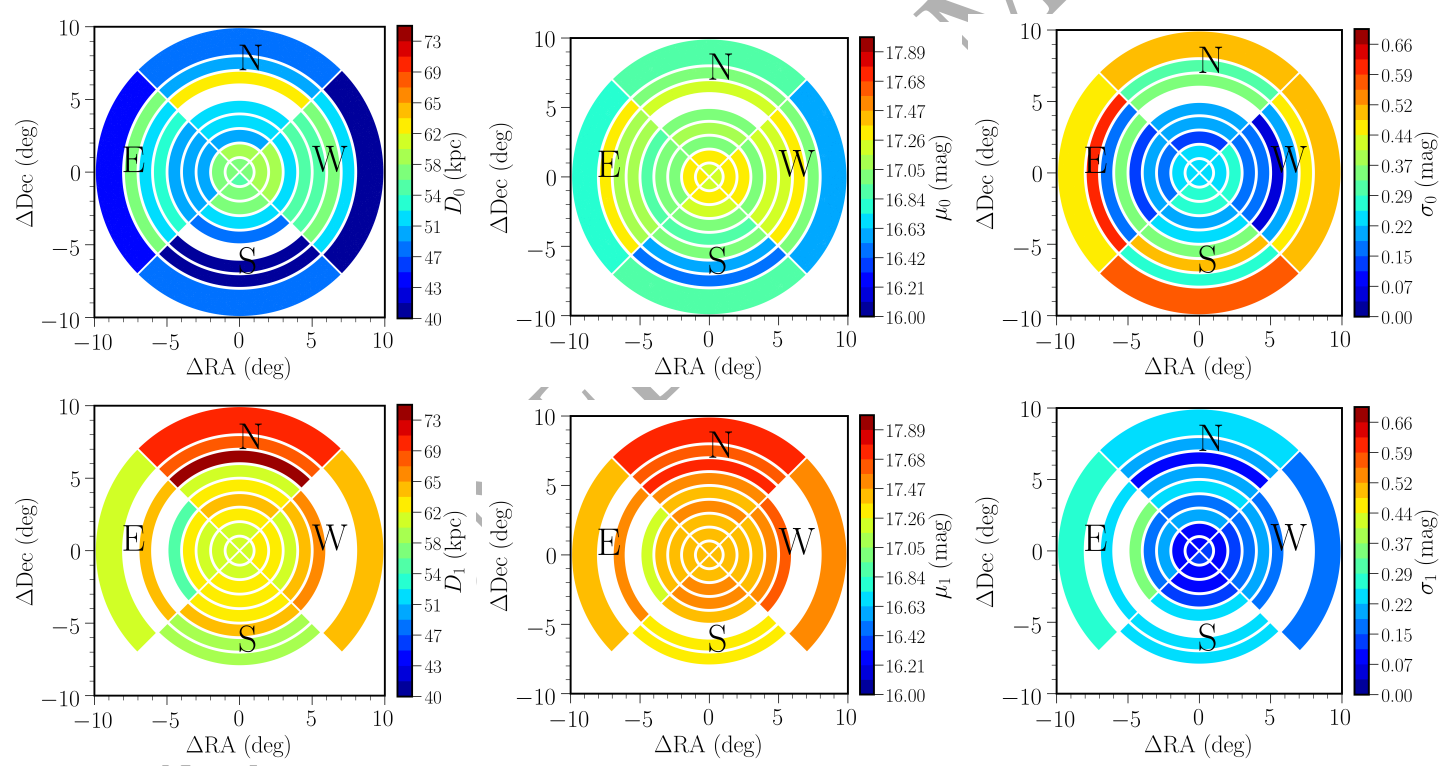

Figure 14. Two-dimensional maps of the distance (left), mean brightness (middle) and sigma (right) of the bright (top) and faint RC components (bottom) in all four sectors, N, E, S and W out to $10^{\circ}$ from the centre of the SMC.

(Taylor 2005) as well as the following open-source Python packages: matplotlib (Hunter 2007), NumPy (Oliphant 2015), pandas (MeKinney 2010), SciPy (Jones et al. 2001).

\section{DATA AVAILABILITY}

The mean magnitudes, spread in brightness and distances of RC stars in different sectors are provided in Table 3. The VMC data were released as part of Data Release 5 (DR5) of the VMC survey (see https://www. eso.org/sci/publications/ announcements/sciann17232.html). VHS observations obtained until 2017 March 30 were released as part of DR5 of the 
Table 3. Gaussian parameters and the reduced $\chi^{2}$ values of the profile fits to the luminosity function of the RC stars in different areas.

\begin{tabular}{|c|c|c|c|c|c|c|c|c|c|c|c|}
\hline Region & $\begin{array}{r}\mu_{0} \\
(\mathrm{mag})\end{array}$ & $\begin{array}{r}\sigma_{0} \\
(\mathrm{mag})\end{array}$ & $\begin{array}{r}N_{0} \\
\text { (counts) }\end{array}$ & $\begin{array}{r}D_{0} \\
(\mathrm{kpc})\end{array}$ & $\begin{array}{r}\sigma_{D 0} \\
(\mathrm{kpc})\end{array}$ & $\begin{array}{r}\mu_{1} \\
(\mathrm{mag})\end{array}$ & $\begin{array}{r}\sigma_{1} \\
(\operatorname{mag})\end{array}$ & $\begin{array}{r}N_{1} \\
\text { (counts) }\end{array}$ & $\begin{array}{r}D_{1} \\
(\mathrm{kpc})\end{array}$ & $\begin{array}{r}\sigma_{D 1} \\
(\mathrm{kpc})\end{array}$ & $\chi^{2}$ \\
\hline $0-1^{\circ} \mathrm{N}$ & $17.26 \pm 0.01$ & 0.25 & $1083 \pm 83$ & $57.5 \pm 0.5$ & 6.62 & $17.40 \pm 0.01$ & 0.10 & $1312 \pm 90$ & $61.3 \pm 0.4$ & 2.97 & 2.93 \\
\hline $0-1^{\circ} \mathrm{E}$ & $17.27 \pm 0.01$ & 0.25 & $1282 \pm 146$ & $57.9 \pm 0.6$ & 6.77 & $17.40 \pm 0.01$ & 0.10 & $1460 \pm 155$ & $61.3 \pm 0.5$ & 2.97 & 6.93 \\
\hline $0-1^{\circ} \mathrm{S}$ & $17.21 \pm 0.03$ & 0.24 & $947 \pm 147$ & $56.3 \pm 1.0$ & 6.30 & $17.40 \pm 0.01$ & 0.12 & $1607 \pm 177$ & $61.5 \pm 0.5$ & 3.34 & 6.62 \\
\hline $0-1^{\circ} \mathrm{W}$ & $17.25 \pm 0.02$ & 0.24 & $1094 \pm 147$ & $57.2 \pm 0.7$ & 6.45 & $17.40 \pm 0.01$ & 0.12 & $1444 \pm 155$ & $61.3 \pm 0.5$ & 3.34 & 5.22 \\
\hline $1-2^{\circ} \mathrm{N}$ & $17.31 \pm 0.01$ & 0.25 & $1242 \pm 86$ & $58.7 \pm 0.5$ & 6.89 & $17.41 \pm 0.01$ & 0.10 & $761 \pm 87$ & $61.5 \pm 0.5$ & 2.98 & 2.48 \\
\hline $1-2^{\circ} \mathrm{E}$ & $17.26 \pm 0.01$ & 0.25 & $1959 \pm 136$ & $57.6 \pm 0.5$ & 6.58 & $17.40 \pm 0.01$ & 0.10 & $1691 \pm 132$ & $61.5 \pm 0.5$ & 2.89 & 4.83 \\
\hline $1-2^{\circ} \mathrm{S}$ & $17.26 \pm 0.01$ & 0.27 & $1703 \pm 92$ & $57.6 \pm 0.5$ & 7.16 & $17.43 \pm 0.01$ & 0.10 & $2448 \pm 163$ & $62.1 \pm 0.4$ & 2.87 & 6.51 \\
\hline $1-2^{\circ} \mathrm{W}$ & $17.32 \pm 0.01$ & 0.24 & $1658 \pm 127$ & $59.2 \pm 0.5$ & 6.64 & $17.44 \pm 0.01$ & 0.10 & $1768 \pm 134$ & $62.5 \pm 0.4$ & 2.94 & 4.24 \\
\hline $2-3^{\circ} \mathrm{N}$ & $16.98 \pm 0.02$ & 0.14 & $168 \pm 20$ & $50.6 \pm 0.7$ & 3.19 & $17.44 \pm 0.01$ & 0.21 & $650 \pm 15$ & $62.5 \pm 0.5$ & 6.15 & \\
\hline $2-3^{\circ} \mathrm{E}$ & $16.95 \pm 0.03$ & 0.18 & $418 \pm 64$ & $49.8 \pm 0.9$ & 4.08 & $17.38 \pm 0.02$ & 0.22 & $904 \pm 238$ & $60.7 \pm 0.7$ & 6.14 & 1.65 \\
\hline $2-3^{\circ} \mathrm{S}$ & $17.26 \pm 0.01$ & 0.29 & $692 \pm 29$ & $57.4 \pm 0.5$ & 7.66 & $17.47 \pm 0.01$ & 0.11 & $844 \pm 40$ & $63.5 \pm 0.4$ & 3.19 & 1.49 \\
\hline $2-3^{\circ} \mathrm{W}$ & $17.35 \pm 0.01$ & 0.28 & $582 \pm 54$ & $60.1 \pm 0.5$ & 7.88 & $17.46 \pm 0.01$ & 0.11 & $829 \pm 57$ & $63.2 \pm 0.5$ & & \\
\hline $3-4^{\circ} \mathrm{N}$ & $17.08 \pm 0.04$ & 0.19 & $153 \pm 14$ & $52.9 \pm 1.1$ & 4.72 & $17.52 \pm 0.04$ & 0.18 & $143 \pm 17$ & $64.8 \pm 1.3$ & 5.31 & 1.36 \\
\hline $3-4^{\circ} \mathrm{E}$ & $16.99 \pm 0.01$ & 0.19 & $520 \pm 17$ & $50.8 \pm 0.5$ & 4.44 & $17.45 \pm 0.02$ & 0.16 & $356 \pm 21$ & $62.7 \pm 0.6$ & 4.56 & 1.57 \\
\hline $3-4^{\circ} \mathrm{S}$ & $17.04 \pm 0.01$ & 0.20 & $195 \pm 8$ & $52.0 \pm 0.5$ & 4.88 & $17.49 \pm 0.01$ & 0.12 & $196 \pm 12$ & $63.8 \pm 0.5$ & 3.44 & 1.08 \\
\hline $3-4^{\circ} \mathrm{W}$ & $17.01 \pm 0.08$ & 0.16 & $48 \pm 12$ & $51.4 \pm 2.0$ & 3.78 & $17.42 \pm 0.02$ & 0.17 & $240 \pm 12$ & $62.0 \pm 0.7$ & 4.79 & 1.38 \\
\hline $4-5^{\circ} \mathrm{N}$ & $17.02 \pm 0.12$ & 0.19 & $111 \pm 73$ & $51.6 \pm 2.9$ & 4.46 & $17.45 \pm 0.06$ & 0.24 & & $62.7 \pm 1.7$ & 7.10 & 1.60 \\
\hline $4-5^{\circ} \mathrm{E}$ & $16.94 \pm 0.01$ & 0.13 & $350 \pm 37$ & $49.6 \pm 0.4$ & 3.08 & $17.21 \pm 0.02$ & 0.35 & $435 \pm 28$ & $56.2 \pm 0.7$ & 9.05 & 1.31 \\
\hline $4-5^{\circ} \mathrm{S}$ & $16.90 \pm 0.08$ & 0.22 & $147 \pm 29$ & $48.8 \pm 1.9$ & 5.05 & $17.44 \pm 0.07$ & 0.25 & $200 \pm 28$ & $62.4 \pm 2.0$ & 7.15 & 2.75 \\
\hline $4-5^{\circ} \mathrm{W}$ & $17.16 \pm 0.14$ & 0.16 & $53 \pm 42$ & $55.0 \pm 3.6$ & 4.12 & $17.53 \pm 0.10$ & 0.20 & $117 \pm 26$ & $65.3 \pm 2.9$ & 6.16 & 1.66 \\
\hline $5-6^{\circ} \mathrm{N}$ & - & - & - & - & - & $17.40 \pm 0.01$ & 0.32 & $200 \pm 7$ & $61.3 \pm 0.5$ & 9.14 & 0.99 \\
\hline $5-6^{\circ} \mathrm{E}$ & $17.15 \pm 0.02$ & 0.35 & $146 \pm 7$ & $54.7 \pm 0.6$ & 8.88 & - & & - & - & - & 1.37 \\
\hline $5-6^{\circ} \mathrm{S}$ & $17.02 \pm 0.09$ & 0.35 & $101 \pm 9$ & $51.5 \pm 2.3$ & 8.22 & 0.07 & 0.19 & $47 \pm 27$ & $65.5 \pm 2.1$ & 5.70 & 1.42 \\
\hline $5-6^{\circ} \mathrm{W}$ & $16.83 \pm 0.19$ & 0.38 & $23 \pm 5$ & $47.2 \pm 4.1$ & 8.27 & $17.52 \pm 0.06$ & 0.16 & $35 \pm 12$ & $64.8 \pm 1.5$ & 4.80 & 4.62 \\
\hline $6-7^{\circ} \mathrm{N}$ & $17.47 \pm 0.03$ & 0.35 & $65 \pm 5$ & $63.5 \pm 0.9$ & 10.13 & $17.79 \pm 0.04$ & 0.10 & $23 \pm 9$ & $73.4 \pm 1.4$ & 3.29 & 0.99 \\
\hline $6-7^{\circ} \mathrm{E}$ & $17.05 \pm 0.07$ & 0.17 & $33 \pm 8$ & $52.3 \pm 1.7$ & 4.19 & $17.54 \pm 0.08$ & 0.22 & $37 \pm 5$ & $65.3 \pm 2.4$ & 6.70 & 0.79 \\
\hline $6-7^{\circ} \mathrm{S}$ & $16.54 \pm 0.28$ & 0.50 & $19 \pm 3$ & $41.3 \pm 5.3$ & & $17.32 \pm 0.04$ & 0.26 & $50 \pm 11$ & $59.0 \pm 1.2$ & 6.98 & 1.29 \\
\hline $6-7^{\circ} \mathrm{W}$ & $17.30 \pm 0.04$ & 0.19 & $14 \pm 3$ & $47.9 \pm 1.2$ & & - & - & - & - & 5.11 & 1.15 \\
\hline $7-8^{\circ} \mathrm{N}$ & $17.00 \pm 0.32$ & 0.32 & $14 \pm 5$ & +7.5 & 7.64 & $17.67 \pm 0.07$ & 0.22 & $44 \pm 11$ & $69.5 \pm 2.4$ & 6.97 & 2.85 \\
\hline $7-8^{\circ} \mathrm{E}$ & $17.27 \pm 0.07$ & 0.62 & $17 \pm 2$ & & 16.53 & - & - & - & - & - & 1.25 \\
\hline $7-8^{\circ} \mathrm{S}$ & $16.52 \pm 0.07$ & 0.27 & $19 \pm 3$ & $40.9 \pm 1.4$ & 5.16 & $17.34 \pm 0.04$ & 0.23 & $38 \pm 5$ & $59.8 \pm 1.1$ & 6.43 & 1.87 \\
\hline $7-8^{\circ} \mathrm{W}$ & $17.03 \pm 0.08$ & 0.45 & $7 \pm 1$ & & 10.77 & - & - & - & - & - & 1.06 \\
\hline $8-10^{\circ} \mathrm{N}$ & $16.92 \pm 0.39$ & 0.50 & $21 \pm 5$ & & 11.47 & $17.70 \pm 0.05$ & 0.22 & $53 \pm 17$ & $70.3 \pm 1.8$ & 7.24 & 3.21 \\
\hline $8-10^{\circ} \mathrm{E}$ & $16.74 \pm 0.62$ & 0.47 & $21 \pm$ & $5.3 \pm 13.0$ & 9.74 & $17.41 \pm 0.12$ & 0.27 & $39 \pm 28$ & $61.8 \pm 3.1$ & 7.81 & 1.81 \\
\hline $8-10^{\circ} \mathrm{S}$ & $16.91 \pm 0.06$ & 0.56 & & $48.9 \pm 1.4$ & 12.71 & - & - & - & - & - & 2.43 \\
\hline $8-10^{\circ} \mathrm{W}$ & $16.56 \pm 0.13$ & 0.50 & & $41.6 \pm 2.6$ & 9.65 & $17.50 \pm 0.06$ & 0.16 & $28 \pm 10$ & $64.2 \pm 1.8$ & 4.8 & 4.18 \\
\hline
\end{tabular}

VHS survey (see https://www.eso.org/sci/publications/ announcements/sciann17290.html), whereas VHS observations obtained until 2017 September 30 will be released soon.

\section{REFERENCES}

Abbott T. M. C., et al., 2018, The Astrophysical Journal Supplement Series, 239,18

Alves D. R., 2000, ApJ, 539, 732

Bagheri G., Cioni M. R. L., Napiwotzki R., 2013, A\&A, 551, A78

Belokurov V. A., Erkal D., 2019, MNRAS, 482, L9

Belokurov V., Erkal D., Deason A. J., Koposov S. E., De Angeli F., Evans D. W., Fraternali F., Mackey D., 2017, MNRAS, 466, 4711

Besla G., Kallivayalil N., Hernquist L., Robertson B., Cox T. J., van der Marel R. P., Alcock C., 2007, ApJ, 668, 949
Carrera R., Conn B. C., Noël N. E. D., Read J. I., López Sánchez Á. R., 2017, MNRAS, 471, 4571

Choi Y., et al., 2018a, ApJ, 866, 90

Choi Y., et al., 2018b, ApJ, 866, 90

Cioni M. R. L., Habing H. J., Israel F. P., 2000, A\&A, 358, L9

Cioni M.-R. L., Marquette J.-B., Loup C., Azzopardi M., Habing H. J., Lasserre T., Lesquoy E., 2001, A\&A, 377, 945

Cioni M.-R. L., et al., 2011, A\&A, 527, A116

Clementini G., et al., 2019, A\&A, 622, A60

Cross N. J. G., et al., 2012, A\&A, 548, A119

Dalton G. B., et al., 2006, in McLean I. S., Iye M., eds, Vol. 6269, Groundbased and Airborne Instrumentation for Astronomy. SPIE, pp 314 - 323, doi:10.1117/12.670018, https://doi.org/10.1117/12.670018

Deason A. J., Belokurov V., Erkal D., Koposov S. E., Mackey D., 2017, MNRAS, 467, 2636

Diaz J. D., Bekki K., 2012, ApJ, 750, 36 
Dobbie P. D., Cole A. A., Subramaniam A., Keller S., 2014, MNRAS, 442,1663

Drlica-Wagner A., et al., 2016, ApJ, 833, L5

El Youssoufi D., et al., 2019, MNRAS, 490, 1076

Emerson J., McPherson A., Sutherland W., 2006, The Messenger, 126, 41

Gaia Collaboration et al., 2016, A\&A, 595, A2

Gaia Collaboration et al., 2018, A\&A, 616, A12

Gaia Collaboration et al., 2020, arXiv e-prints, p. arXiv:2012.01771

Girardi L., 2016, ARA\&A, 54, 95

González-Fernández C., et al., 2018, MNRAS, 474, 5459

Groenewegen M. A. T., 2008, A\&A, 488, 935

Harris J., 2007, ApJ, 658, 345

Hawkins K., Leistedt B., Bovy J., Hogg D. W., 2017, MNRAS, 471, 722

Hindman J. V., Kerr F. J., McGee R. X., 1963, Australian Journal of Physics, 16,570

Hunter J. D., 2007, Computing in Science and Engineering, 9, 90

Irwin M. J., et al., 2004, in Quinn P. J., Bridger A., eds, Proc. SPIEVol. 5493, Optimizing Scientific Return for Astronomy through Information Technologies. pp 411-422, doi:10.1117/12.551449

Jacyszyn-Dobrzeniecka A. M., et al., 2017, Acta Astron., 67, 1

Jacyszyn-Dobrzeniecka A. M., et al., 2020a, ApJ, 889, 25

Jacyszyn-Dobrzeniecka A. M., et al., 2020b, ApJ, 889, 26

Jones E., Oliphant T. E., Peterson P., et al., 2001, SciPy: Open source scientific tools for Python, http: //www . scipy.org/

Kallivayalil N., van der Marel R. P., Alcock C., Axelrod T., Cook K. H., Drake A. J., Geha M., 2006, ApJ, 638, 772

Laney C. D., Joner M. D., Pietrzyński G., 2012, MNRAS, 419, 1637

Mackey A. D., Koposov S. E., Erkal D., Belokurov V., Da Costa G. S., Gómez F. A., 2016, MNRAS, 459, 239

Mackey A. D., Koposov S. E., Da Costa G. S., Belokurov V., Erkal D., Fraternali F., McClure-Griffiths N. M., Fraser M., 2017, MNRAS, 472, 2975

Mackey D., Koposov S., Da Costa G., Belokurov V., Erkal D., Kuzma P., 2018, ApJ, 858, L21

Martin D. C., et al., 2005, ApJ, 619, L1

Martínez-Delgado D., et al., 2019, A\&A, 631, A98

Massana P., et al., 2020, MNRAS, 498, 1034

Mathewson D. S., Cleary M. N., Murray J. D., 1974, ApJ, 190, 291

McClure-Griffiths N. M., et al., 2008, ApJ, 673, L143

McKinney W., 2010, in van der Walt S., Millman J., eds, Proceedings of the 9th Python in Science Conference. p. 51

McMahon R. G., Banerji M., Gonzalez E., Koposov S. E., Bejar V. J., Lodieu N., Rebolo R., VHS Collaboration 2013, The Messenger, 154, 35

Muraveva T., et al., 2018, MNRAS, 473, 3131

Neugebauer G., Leighton R. B., 1969, Two-micron sky survey. A preliminary catalogue

Nidever D. L., Majewski S. R., Butler Burton W., Nigra L., 2010, ApJ, 723,1618

Nidever D. L., Monachesi A., Bell E. F., Majewski S. R., Muñoz R. R., Beaton R. L., 2013, ApJ, 779, 145

Nidever D. L., et al., 2017, AJ, 154, 199

Nikolaev S., Weinberg M. D., 2000, ApJ, 542, 804

Noël N. E. D., Conn B. C., Read J. I., Carrera R., Dolphin A., Rix H. W., 2015, MNRAS, 452, 4222

Oliphant T. E., 2015, Guide to NumPy, CreateSpace Independent Publishing Platform, 2nd ed.

Olsen K. A. G., Salyk C., 2002, AJ, 124, 2045

Olsen K. A. G., Zaritsky D., Blum R. D., Boyer M. L., Gordon K. D., 2011, ApJ, 737, 29

Omkumar A. O., et al., 2021, MNRAS, 500, 2757

Pieres A., et al., 2017, MNRAS, 468, 1349

Putman M. E., et al., 1998, Nature, 394, 752

Ripepi V., et al., 2017, MNRAS, 472, 808

Rubele S., et al., 2018, MNRAS, 478, 5017

Ruiz-Dern E., Babusiaux C., Arenou F., Turon C., Lallement R., 2018, A\&A, 609, A116

Salaris M., Girardi L., 2002, MNRAS, 337, 332

Salem M., Besla G., Bryan G., Putman M., van der Marel R. P., Tonnesen S., 2015, ApJ, 815, 77
Schlafly E. F., Finkbeiner D. P., 2011, ApJ, 737, 103

Schlegel D. J., Finkbeiner D. P., Davis M., 1998, ApJ, 500, 525

Schmidt T., et al., 2020, A\&A, 641, A134

Scowcroft V., Freedman W. L., Madore B. F., Monson A., Persson S. E., Rich J., Seibert M., Rigby J. R., 2016, ApJ, 816, 49

Shapley H., 1940, Harvard Coll. Obs. Bull., 914, 8

Skowron D. M., et al., 2014, ApJ, 795, 108

Skrutskie M. F., et al., 2006, AJ, 131, 1163

Subramanian S., Subramaniam A., 2010, A\&A, 520, A24

Subramanian S., Subramaniam A., 2012, ApJ, 744, 128

Subramanian S., Subramaniam A., 2013, A\&A, 552, A144

Subramanian S., et al., 2017, MNRAS, 467, 2980

Sutherland, Will et al., 2015, A\&A, 575, A25

Tatton B., 2018, PhD thesis, Keele University, http://eprints.keele. ac.uk/5587/

Tatton B. L., et al., 2013, A\&A, 554, A33

Tatton B. L., et al., 2020, arXiv e-prints, p. arXiv:2012.12288

Taylor M. B., 2005, in Shopbell P., Britton M., Ebert R., eds, Astronomical Society of the Pacific Conference Series Vol. 347, Astronomical Data Analysis Software and Systems XIV. p. 29

Wang J., Hammer F., Yang Y., Ripepi V., Cioni M.-R. L., Puech M., Flores H., 2019, MNRAS, 486, 5907

Zaritsky D., Harris J., Grebel E. K., Thompson I. B., 2000, ApJ, 534, L53

Zhao H., Evans N. W., 2000, ApJ, 545, L35

Zivick P., et al., 2019, ApJ, 874, 78

Zivick P., Kallivayalil N., van der Marel R. P., 2020, arXiv e-prints, p. arXiv:2011.02525

de Grijs R., Bono G., 2014a, AJ, 148, 17

de Grijs R., Bono G., 2014b, AJ, 148, 17

de Grijs R., Bono G., 2015, AJ, 149, 179

de Vaucouleurs G., 1955, AJ, 60,126

de Vaucouleurs G., Freeman K. C., 1972, Vistas Astron., 14, 163

van der Marel R. P., 2001, AJ, 122, 1827

\section{APPENDIX A: HESS DIAGRAMS AND LUMINOSITY FUNCTIONS OF RC STARS \\ APPENDIX B: ALTERNATIVE CARDINAL DIVISION FOR RC STARS}

We explore an alternative cardinal division of the spatial distribution of stars around the SMC. The galaxy is still divided into circular annuli of radii $1^{\circ}, 2^{\circ}, 3^{\circ}, 4^{\circ}, 5^{\circ}, 6^{\circ}, 7^{\circ}, 8^{\circ}$ and $10^{\circ}$ from the centre and each annulus into four sectors: North-East (NE), North-West (NW), South-East (E) and South-West (W), see Figure B1. These four regions are $45^{\circ}$ offset counter-clockwise from the sectors adopted in Sect. 4. As in the previous analysis, we study their NIR $\left(J-K_{\mathrm{S}}\right.$, $K_{\mathrm{S}}$ ) CMD diagrams and RC luminosity functions (Figure B2). We only show sectors where a faint component which might be related to the Counter Bridge is detected. Figure B3 shows how the cardinal divisions explored in this study map onto the morphological features. 

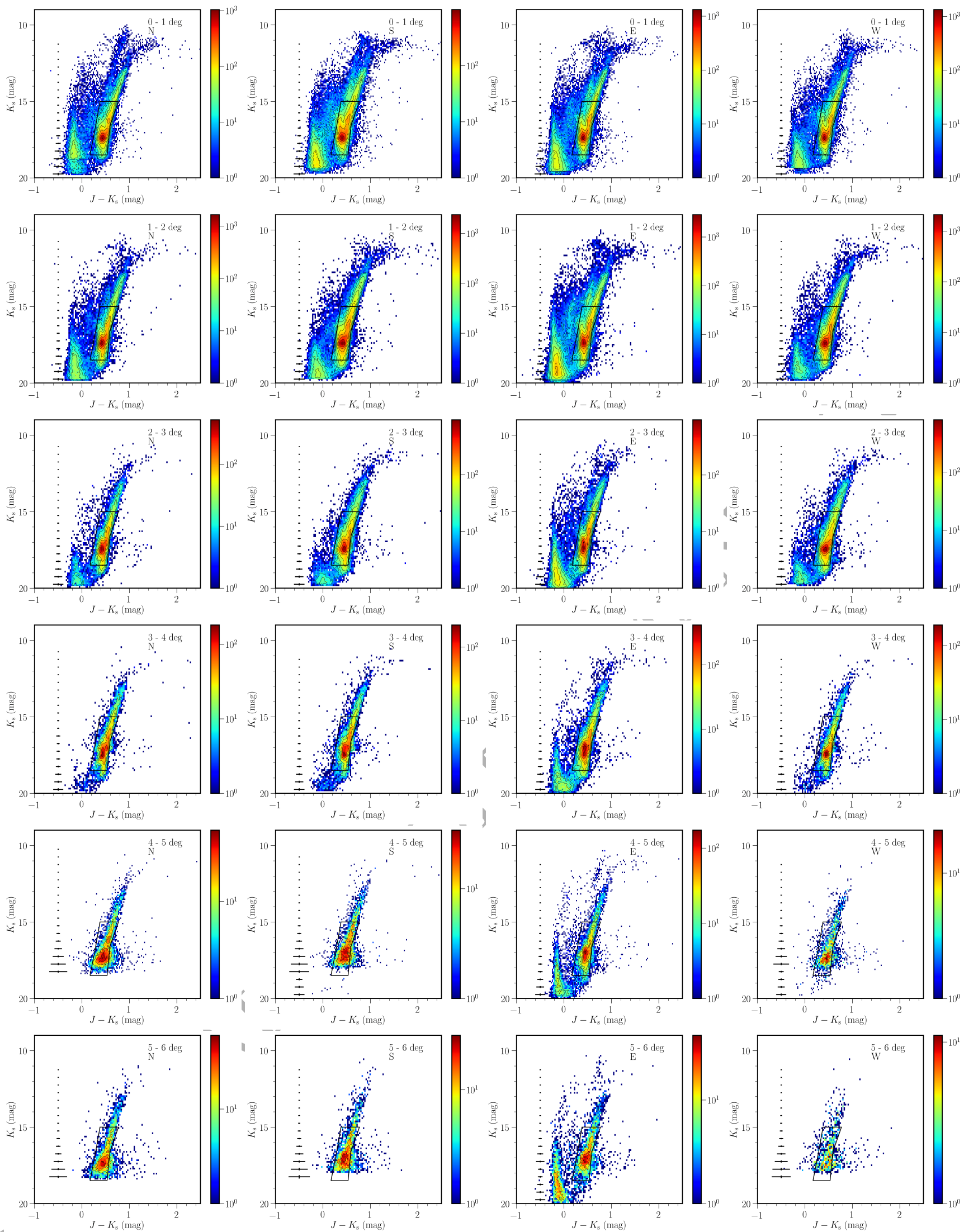

Figure A1. NIR $\left(J-K_{\mathrm{s}}, K_{\mathrm{S}}\right)$ Hess diagrams of different SMC regions. The colour scale indicates the stellar density on a logarithmic scale per bin. The bin size is $0.027 \times 0.110 \mathrm{deg}^{2}$ and the black box limits the region defined to study the distribution of RC stars. 
Stellar substructures in the periphery of the MCs
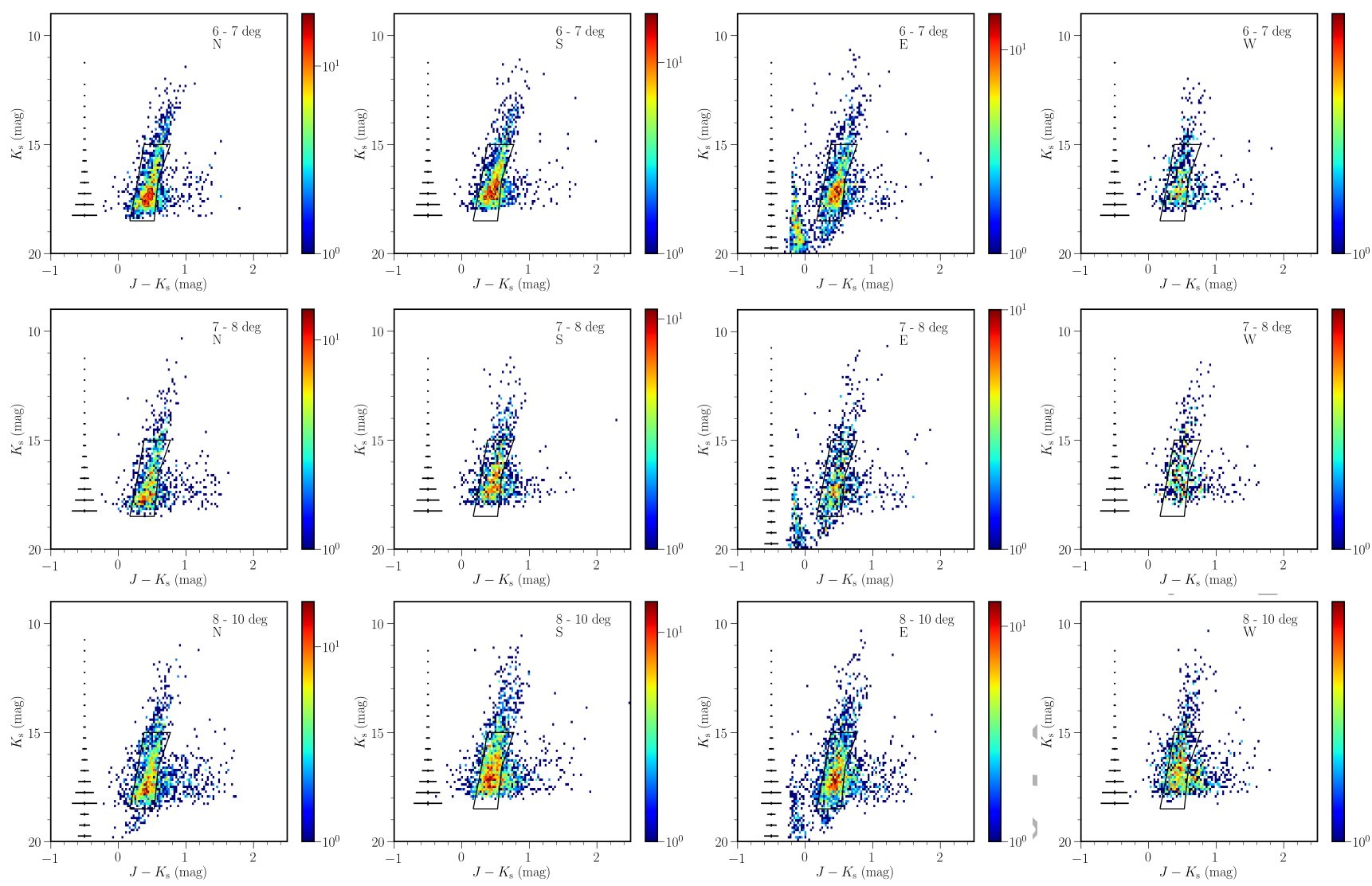

Figure A1. (continued) 

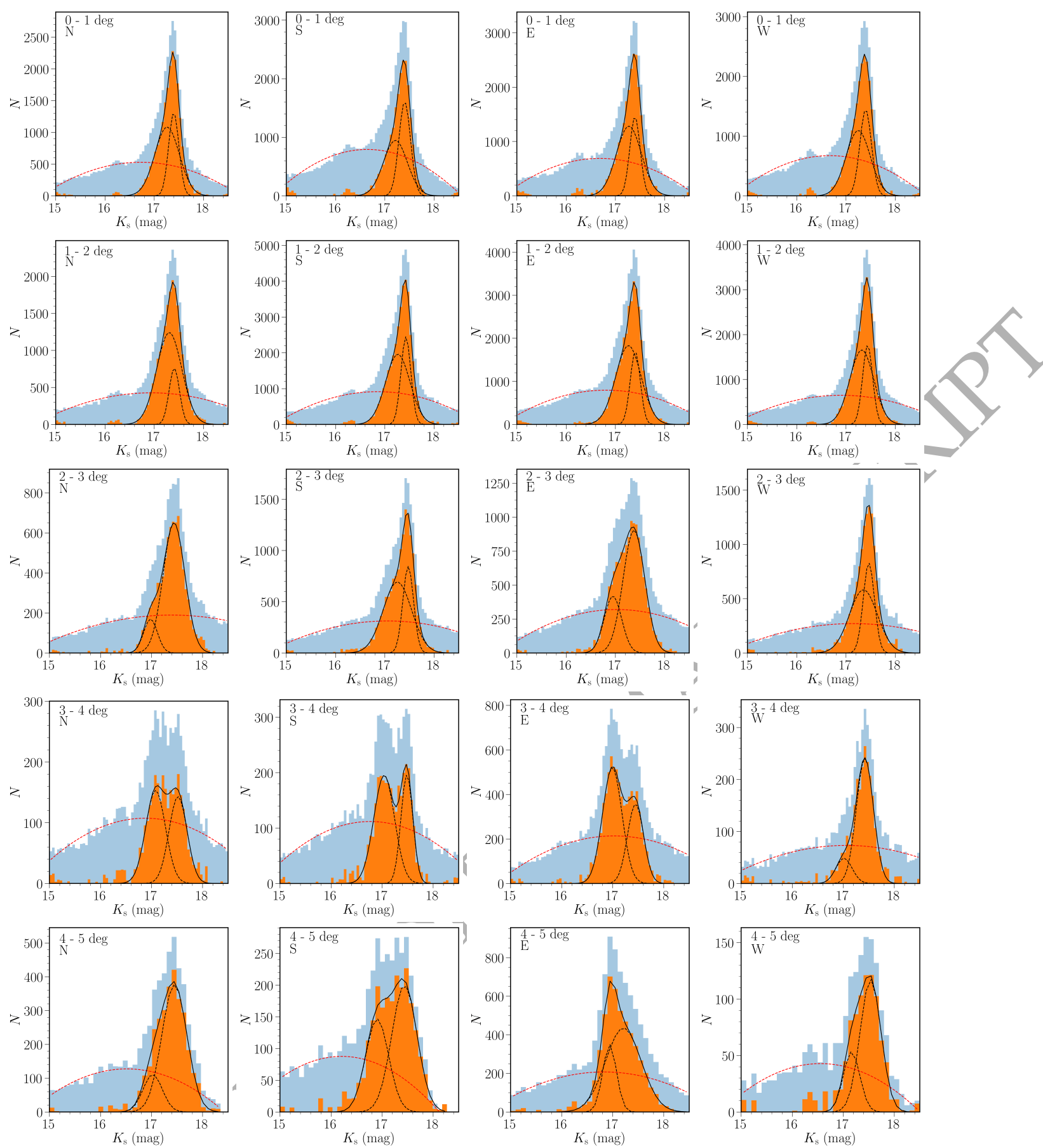

Figure A2. Luminosity function of the RC stars in different SMC regions. The blue histograms show the observed luminosity functions, whereas orange histograms show the distributions after subtracting the RGB components and the continuous lines show the total fits to these distributions while the dashed lines represent the separate components of the fits. 

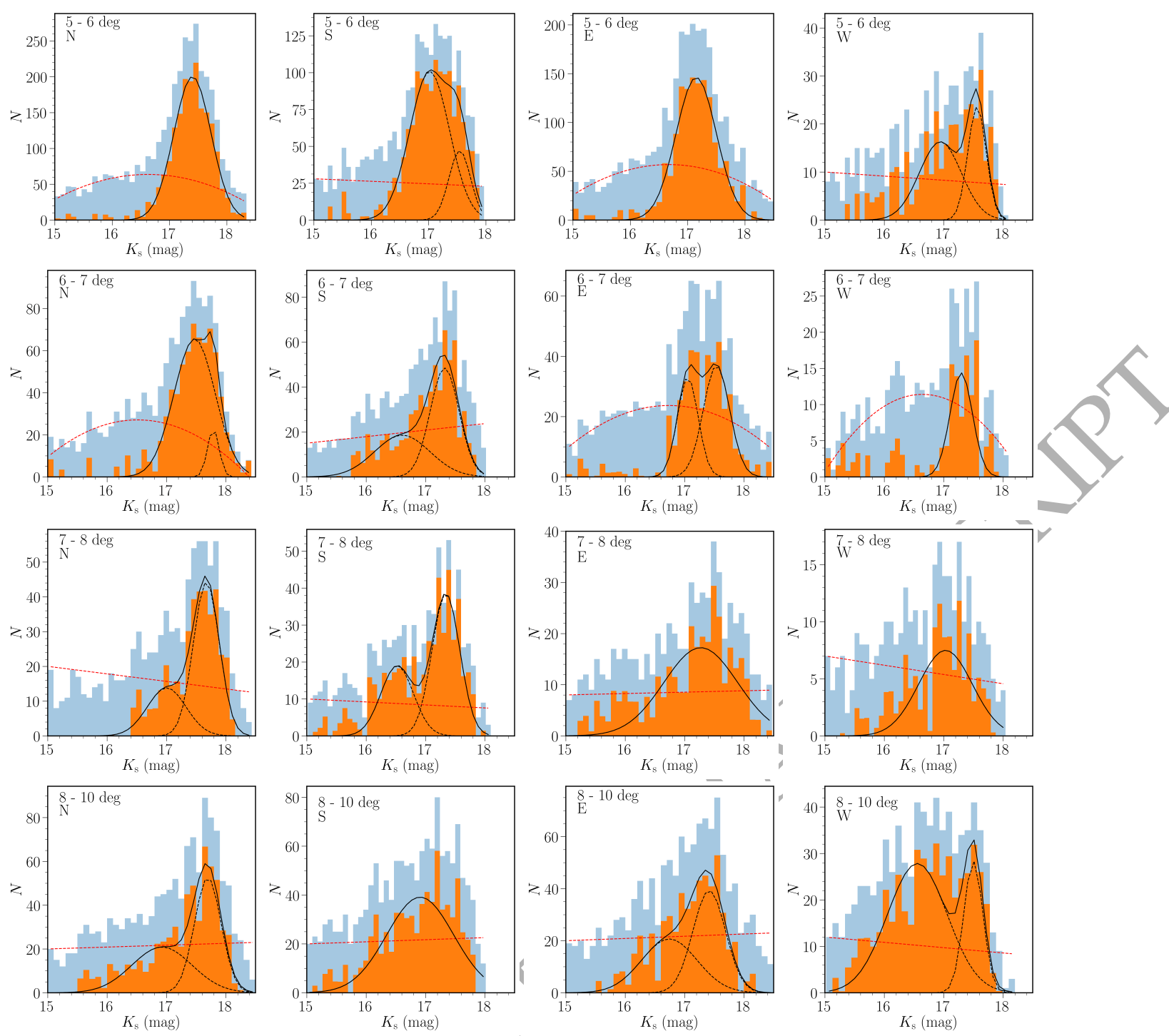

Figure A2. (continued) 


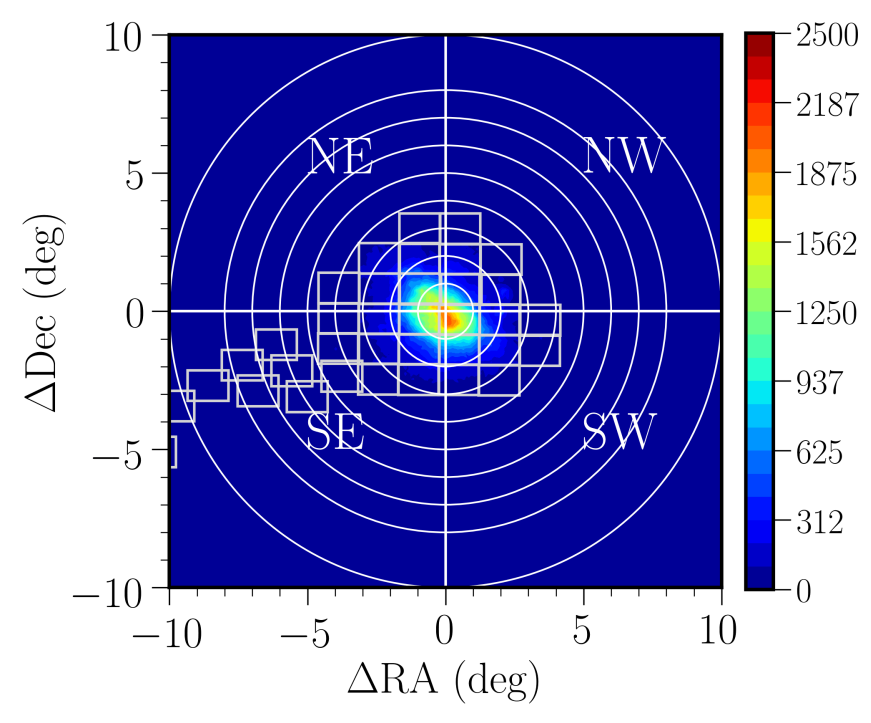

Figure B1. Spatial distribution of stars around the SMC and plotted annuli from $0^{\circ}$ to $10^{\circ}$ further divided into 4 regions: NE, NW, SE and SW. VMC tiles are also shown.
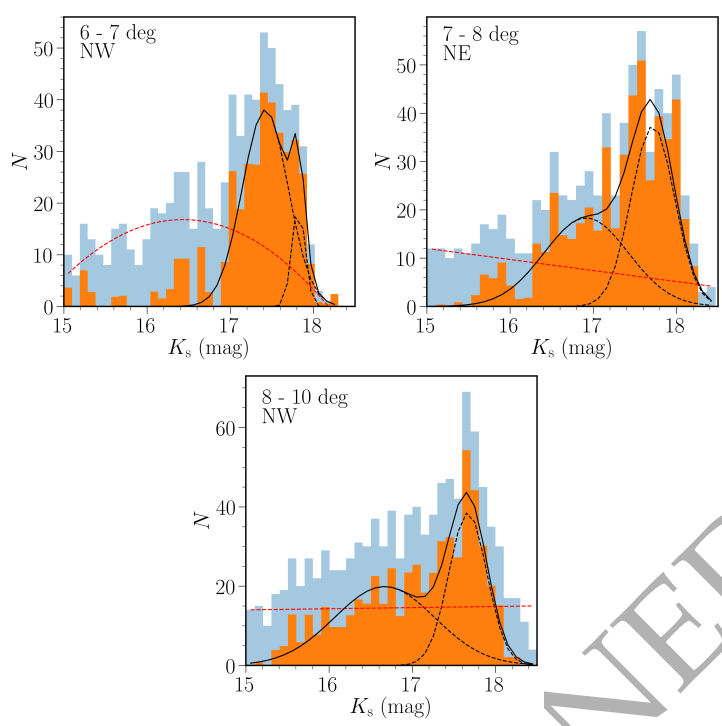

Figure B2. Luminosity function of the RC stars in different regions. Each panel shows the luminosity function of the regions discussed in Sect. 4 and Appendix B. The blue histograms show the observed luminosity functions, whereas orange histograms show the distributions after subtracting the RGB components and the continuous lines show the total fits to these distributions whereas the dashed lines represent the separate components of the fits.
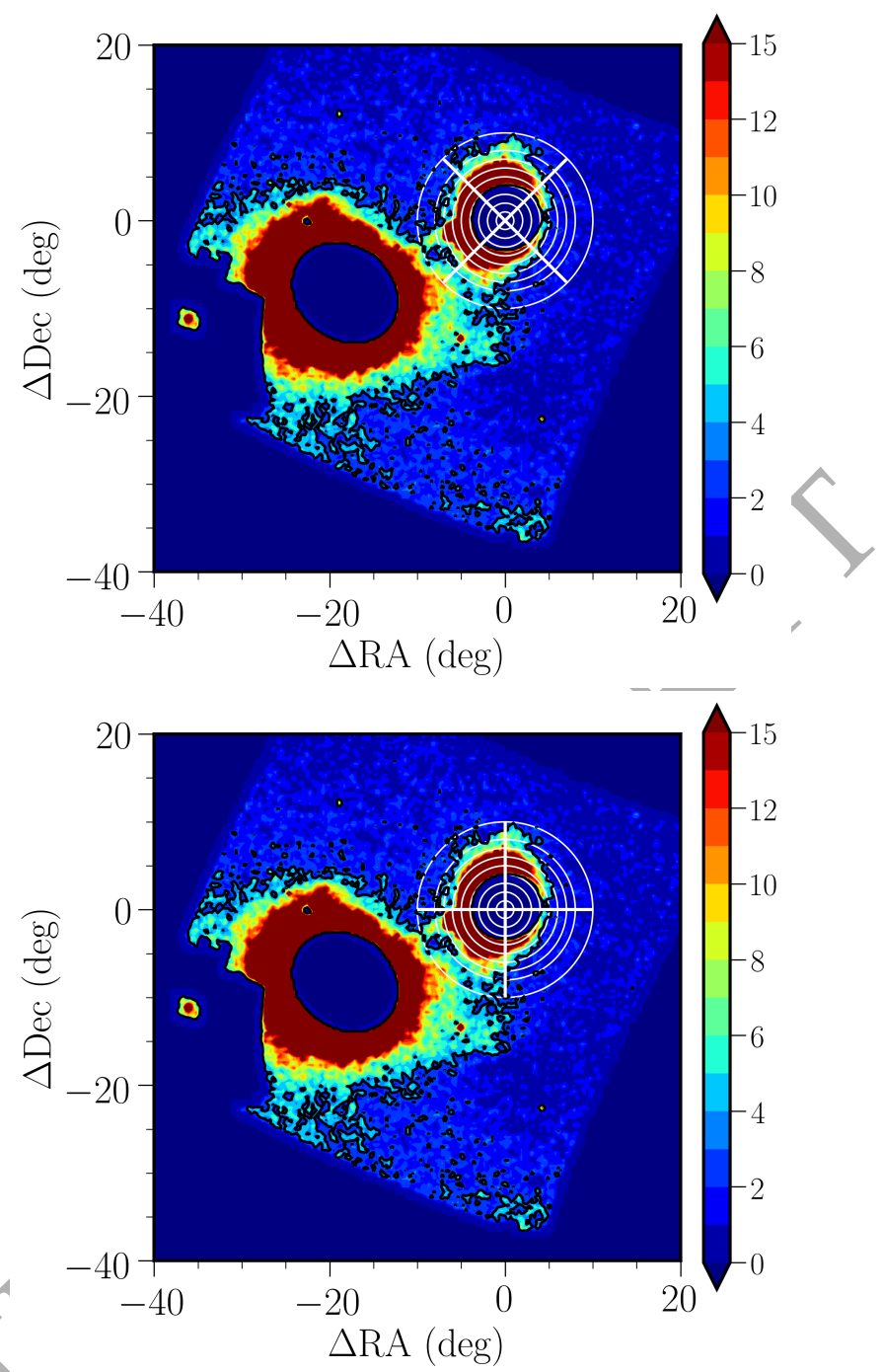

Figure B3. Spatial division of the SMC area into sectors and rings as in Fig. 6 (top) and Fig. B1 (bottom) superimposed on the density of young and old stars as in Fig. 9, depicting the morphological features analysed in this study. 